\title{
13. UPPER CRETACEOUS CYCLIC SEDIMENTS FROM HOLE 762C, EXMOUTH PLATEAU, NORTHWEST AUSTRALIA ${ }^{1}$
}

\author{
Zehui Huang, ${ }^{2}$ Ron Boyd, ${ }^{2}$ and Suzanne O'Connell ${ }^{3}$
}

\begin{abstract}
Well-developed Campanian to Maestrichtian pelagic cyclic sediments were recovered from Hole $762 \mathrm{C}$ on the Exmouth Plateau, off northwest Australia, during Ocean Drilling Program Leg 122. The cycles consist of nannofossil chalk (light beds) and clayey nannofossil chalk (dark beds). Both light and dark beds are strongly to moderately bioturbated, alternate on a decimeter scale, and exhibit gradual boundaries. Bioturbation introduces materials from a bed of one color into an underlying bed of another color, indicating that diagenesis is not responsible for the cyclicity. Differences in composition between the light and dark beds, revealed by calcium carbonate measurement and X-ray diffraction analysis, together with trace fossil evidence, indicate that the cycles in the sediments are a depositional feature. Diagenetic processes may have intensified the appearance of the cycles.

Spectral analysis was applied to the upper Campanian to lower Maestrichtian cyclic sediments to examine the regularity of the cycles. Power spectra were calculated from time series using Walsh spectral analysis. The most predominant wavelengths of the color cycles are $34-41 \mathrm{~cm}$ and $71-84 \mathrm{~cm}$. With an average sedimentation rate of $1.82 \mathrm{~cm} / \mathrm{k} . \mathrm{y}$. in this interval, we found the time durations of the cycles to be around $41 \mathrm{k} . \mathrm{y}$. and $21 \mathrm{k} . \mathrm{y}$. , respectively, comparable to the obliquity and precession periods of the Earth's rotation, which strongly suggests an orbital origin for the cycles.

On the basis of sedimentological evidence and plate tectonic reconstruction, we propose the following mechanism for the formation of the cyclic sediments from Hole 762C. During the Late Cretaceous, when there was no large-scale continental glaciation, the cyclic variations in insolation, in response to cyclic orbital changes, controlled the alternation of two prevailing climates in the area. During the wetter, equable, and warmer climatic phases under high insolation, more clay minerals and other terrestrial materials were produced on land and supplied by higher runoff to a low bioproductivity ocean, and the dark clayey beds were deposited. During the drier and colder climatic phases under low insolation, fewer clay minerals were produced and put into the ocean, where bioproductivity was increased and the light beds were deposited.
\end{abstract}

\section{INTRODUCTION}

Since Hays et al. (1976) successfully related the climatic cyclicity in Quaternary sediments to the orbital cycles proposed by Milankovitch, many researchers have attempted to relate pre-Pleistocene cyclic sediments, both from land outcrop and from deep-sea drilling, to orbital cycles (e.g., Schwarzacher and Fischer, 1982; Arthur et al., 1984; Cotillon and Rio, 1984; Barron et al., 1985; Weedon, 1986; Herbert and Fischer, 1986; Schwarzacher, 1987; Hart, 1987; Bottjer et al., 1986; Research on Cretaceous Cycles (R.O.C.C.) Group, 1986; Ogg et al., 1987; Jarrard and Arthur, 1989) with various data sets and approaches. However, controversy often followed. The question of diagenetic origin vs. climatic origin of the basal Jurassic cyclic sediments (Blue Lias) of south Britain is one example (Weedon, 1986; Hallam, 1986). This case is the result of the relatively old geological age of the strata, insufficient stratigraphic control, errors in the time scale, and evident diagenetic overprint. Another example is the origin of short-term sea-level fluctuations vs. origin of climatic fluctuations in the Upper Cretaceous marl-limestone sequences in Alabama (King, 1990; Bottjer et al., 1986; R.O.C.C. Group, 1986). The origin of sea-level change here seems plausible because the sequences are the product of shelf sedimentation. The other reason for questioning the conclusion of the

\footnotetext{
1 von Rad, U., Haq, B. U., et al., 1992. Proc. ODP, Sci. Results, 122: College Station, TX (Ocean Drilling Program).

2 Department of Geology, Dalhousie University, Halifax, Nova Scotia B3H $3 \mathrm{~J} 5$, Canada.

3 Department of Earth and Environmental Sciences, Wesleyan University, Middletown, CT 06457, U.S.A.
}

R.O.C.C. Group (1986) on the Alabama Upper Cretaceous marl-limestone sequence is that the sequence contains too few beds for a valid statistical test. Pelagic cyclic sediments younger than Early Cretaceous are better suited for the purpose of testing the theory of orbital origin of sediment cyclicity in pre-Pleistocene time in a quantitative fashion. In this paper we report our study on an Upper Cretaceous cyclic sequence from the Indian Ocean (the Exmouth Plateau). In comparison to Lower Cretaceous cyclic sequences from the Atlantic-Tethyan region (Cotillon and Rio, 1984; Barron et al., 1985; Herbert and Fischer, 1986; Ogg et al., 1987; ten Kate and Sprenger, 1989) and Upper Cretaceous cyclic sequences from the South Atlantic (Herbert and D'Hondt, 1990) and North America (R.O.C.C. Group, 1986), Upper Cretaceous cyclic sequences from the Indian Ocean are less reported.

The Upper Cretaceous cyclic sediments of this study were recovered from Hole $762 \mathrm{C}$, which is located on the western part of the central Exmouth Plateau $\left(19^{\circ} 53.24^{\prime} \mathrm{S}, 112^{\circ} 15.24^{\prime} \mathrm{E}\right)$ in a water depth of $1360 \mathrm{~m}$ and about $260 \mathrm{~km}$ from shore (Fig. 1). The Cretaceous succession from Hole $762 \mathrm{C}$ begins with black to very dark gray silty claystone and clayey siltstone deposited in a shelf-margin prodelta environment during Berriasian to Valanginian time. Uppermost Valanginian to Barremian is represented by a major hiatus (Haq, von Rad, O'Connell, et al., 1990). Lower Aptian sediments are black to dark gray calcareous claystones deposited in a hemipelagic epicontinental shelf environment. The rest of the Cretaceous sediments are mostly pelagic nannofossil chalk and clayey nannofossil chalk with minor claystone. The cyclicity in the form of color band alternation mainly on a decimeter scale begins in Upper Cretaceous sediments (younger than Cenomanian), but is best developed in Cores 122-762C-47X through 


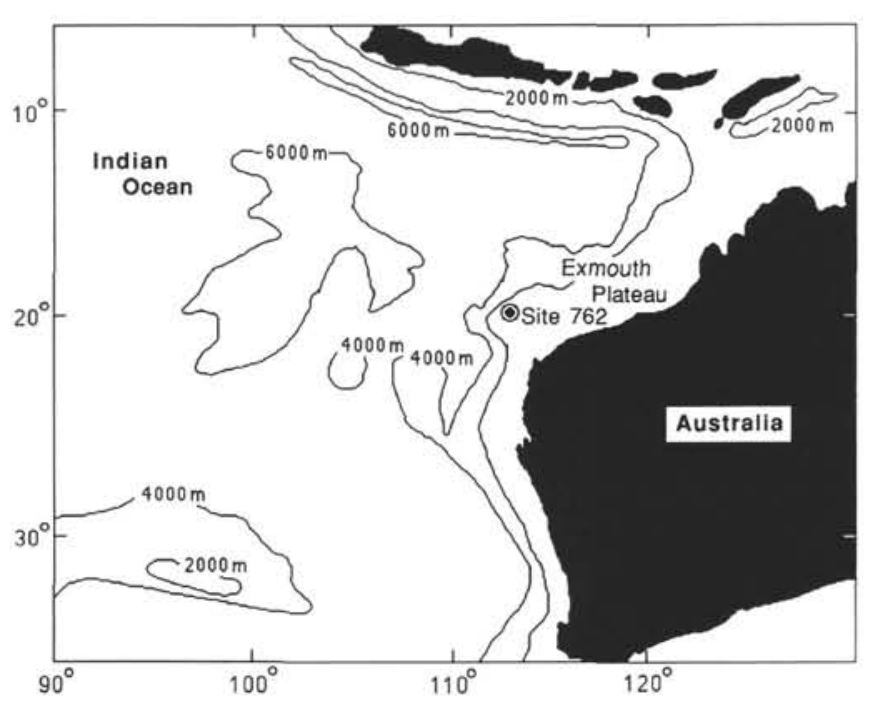

Figure 1. Location of Site 762.

122-762C-56X, 592.5-687.5 meters below seafloor (mbsf). The sediments of this interval (nannofossil chalk) indicate a pelagic environment. The burial history analysis by Gradstein and von Rad (pers. comm., 1990) estimates that the paleowater depth in Late Cretaceous time was at least $550 \mathrm{~m}$. This precludes the possibility that short-term, low-amplitude fluctuations in sea level caused the cyclicity. There is also enough evidence against a diagenetic origin for this sequence. With high core recovery ( $78 \%$ for Cores $122-762 \mathrm{C}-43 \mathrm{X}$ through $-76 \mathrm{X})$, this interval of cyclic sediments provides us with a good opportunity to test the orbital origin of sediment cyclicity in pre-Pleistocene time.

Milankovitch cycles are quasi-periodic variations in the Earth's orbital parameters of precession, obliquity, and eccentricity. Obliquity is the tilt of the polar axis relative to the plane of the orbit, varying from $22^{\circ}$ to $24.5^{\circ}$ with a period $41 \mathrm{k} . \mathrm{y}$. The precession is the change in the seasonal position of the Earth on its way round the ellipse, varying with periods of 19 k.y. and 23 k.y. Eccentricity describes the irregular variation in the ellipticity of the orbit ranging from 0.0005 to 0.0607 , with periodicities of $95 \mathrm{k} . \mathrm{y}, 123 \mathrm{k} . \mathrm{y}$., and 413 k.y. (Berger, 1977). These parameters affect the seasonal and annual latitudinal distribution of solar insolation and long-term global and hemispheric climates (Imbrie and Imbrie, 1980). Obliquity increases its importance with higher latitudes (Berger and Pestiaux, 1984). Precession affected the contrast between summer and winter seasons in each hemisphere. Eccentricity primarily modulates the importance of precession. Because of the long-term change in Earth-Moon distance during geological time, the periods of these orbital elements should be shorter in the geological past than present. Recently Berger and Loutre (1989) calculated the long-term variations of the main orbital parameters during the past 500 m.y., and demonstrated that the periods of these parameters were shorter in the geological past. According to their results, which may be inaccurate for various reasons, the difference in the periods between the present and the Cretaceous is not very significant. For example, the present $41-k$.y. obliquity cycle was about 39.5 k.y. in the Late Cretaceous (around 74 $\mathrm{Ma}$ ). The difference for obliquity periodicity between the Cretaceous and the present is $2,000 \mathrm{yr}$ at most. The difference for precession periodicity is smaller. The precession periodicities were $22.5 \mathrm{k} . \mathrm{y}$. and $18.6 \mathrm{k} . \mathrm{y}$. in the Late Cretaceous (around $74 \mathrm{Ma}$ ). These small differences will not pose a difficulty for testing the Milankovitch theory in Upper Cretaceous strata.
In this study we first examined the features of the cyclic sediments. Sedimentological study indicates that the color alternation is a depositional feature instead of a diagenetic result. Then time series were built on the basis of core inspection. The time series were analyzed with the Walsh spectral analysis method (Beauchamp, 1975) to reveal the periodicity in the sediments, to provide insight into the process of cyclic sedimentation, and to search for the ultimate origin of the cyclicity.

\section{FEATURES OF CYCLIC SEDIMENTS}

Cyclicity in the form of color variation is observed in the Upper Cretaceous pelagic sediments of Cores 122-762C-43X through $-76 \mathrm{X}, 554.5-819.5 \mathrm{mbsf}$. The cyclicity is best developed in Cores 122-762C-47X through - 56X (592.5-687.5 mbsf, upper Campanian to lower Maestrichtian). Figure 2, a photograph of Core $122-762 \mathrm{C}-49 \mathrm{X}$, is an example of upper Campanian to lower Maestrichtian cyclic sediments. The sediments have been classified on the basis of smear slide examination and calcium carbonate abundances using the Ocean Drilling Program (ODP) sediment classification scheme (Haq, von Rad, O'Connell, et al., 1990).

The Upper Cretaceous age in this hole was determined with nannofossils, which are more abundant and better preserved than other minor fossil groups. The nannofossil events observed by Bralower and Siesser (this volume), together with lithological records, indicate a stratigraphically complete Upper Cretaceous in Hole $762 \mathrm{C}$. The biostratigraphy of the interval of interest is shown in Figure 3, following the work by Bralower and Siesser (this volume). The Santonian-Campanian boundary is placed at the first occurrence of Broinsonia parca (774.74 mbsf). The lower Campanian-upper Campanian boundary is placed at the first occurrence of Quadrum gothicum ( $687.80 \mathrm{mbsf})$. The Campanian-Maestrichtian boundary is placed at the last occurrence of Eiffellithus eximius (611.50 mbsf). The lower Maestrichtian-upper Maestrichtian boundary is placed at the first occurrence of Lithraphidites quadratus (578.30 mbsf). There is a hiatus at the Cretaceous-Tertiary boundary. The top of Upper Cretaceous strata is placed at the top of Core 122-762C-43X (554.80 mbsf) where the last Cretaceous species occurs.

In Figure 3, the numeric ages of the stage boundary in millions of years are from the time scale by Kent and Gradstein (1985). For the interval of well-developed cyclicity, we used the lower Maestrichtian-upper Maestrichtian boundary (71 Ma) and the lower Campanian-upper Campanian boundary $(77 \mathrm{Ma})$ as the age control to estimate the average sedimentation rate. The sediment thickness between these two boundaries is $109.50 \mathrm{~m}$ and the duration is $6 \mathrm{~m}$.y. The estimated average sedimentation rate for this interval is 1.82 $\mathrm{cm} / \mathrm{k} . \mathrm{y}$. This average sedimentation rate may contain some errors from both the placement of the age boundaries and from the time scale and is not necessarily constant.

The completeness in both stratigraphy and recovery for the interval of well-developed color alternations enables a detailed study of the nature of cyclic sediments. Because of high gas pressure in the drilling area, the cores were moderately disturbed and slightly expanded, resulting in recovery higher than $100 \%$, but this disturbance is not serious enough to prevent recognizing color variation couplets in most of the cores. Our statistical analysis on the cycles, however, is affected by whole-round sampling for organic geochemistry (OG) analysis and interstitial water (IW) analysis. We are unable to tell if a color variation boundary occurs in a whole-round sample $(25 \mathrm{~cm}$ long for an OG sample and $10 \mathrm{~cm}$ for an IW sample) or, if so, where the boundary is. 


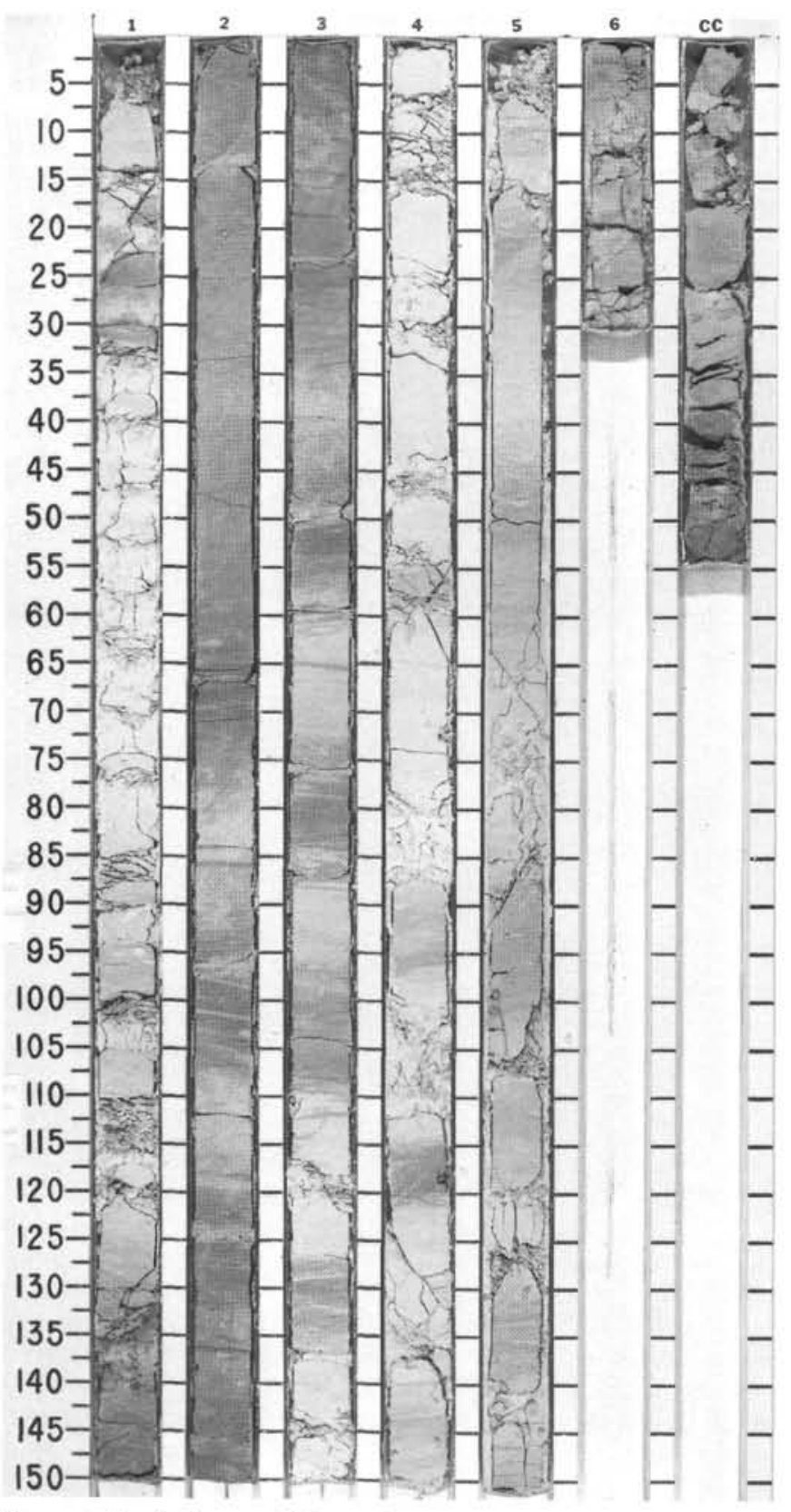

Figure 2. Typical core of Upper Cretaceous pelagic sediments from Hole $762 \mathrm{C}$ displaying alternation of light nannofossil chalk and dark, clayey nannofossil chalk to nannofossil chalk. The boundaries between the light beds and the dark beds are gradual. Core $122-762 \mathrm{C}$ $49 \mathrm{X}$.

\section{General Character of the Cycles}

The 95-m-thick interval of well-developed cyclic sediments displays alternations of light-colored and dark-colored beds (Fig. 2). The color tends to become darker downhole in this interval. Above 603.5 mbsf the light beds are white $(2.5 \mathrm{Y} 8 / 0)$ to very light greenish gray (10Y $8 / 1)$ nannofossil chalk while the dark beds are light greenish gray $(5 \mathrm{GY} 7 / 1)$ clayey nannofossil chalk. Below 603.5 mbsf the light beds are white (10Y $8 / 1)$ to light greenish gray (10Y 7/1) nannofossil chalk and the dark beds are light reddish brown $(5 Y$ $6 / 3)$ to reddish brown (5Y 5/3) clayey nannofossil chalk with some nannofossil chalk.
The thickness of color alternations ranges from 10 to 120 $\mathrm{cm}$ but most of the alternations are on a decimeter scale. Boundaries between the light and dark beds are generally gradual with transitional zones varying from 2 to $12 \mathrm{~cm}$. It is feasible to judge the regularity of the cycles visually.

The thickness of the light and dark beds was measured in Cores $122-762 \mathrm{C}-47 \mathrm{X}$ through $-56 \mathrm{X}$, but beds that are not complete (e.g., whole-round sample intervals) are not included. Light beds $(n=195)$ vary in thickness from 4.0 to 80 $\mathrm{cm}$ with an average of $18 \mathrm{~cm}$ and a standard deviation of 14 $\mathrm{cm}$. Dark beds $(n=194)$ vary from 3.5 to $89 \mathrm{~cm}$ in thickness with an average of $22 \mathrm{~cm}$ and a standard deviation of $18 \mathrm{~cm}$, indicating that the dark beds are thicker and more variable than light beds. In addition, only dark beds show lamination, and where present it is very faint. The lamination is not caused by current activity as there are no ripple cross-bedding or cut and fill features.

\section{Bioturbation}

Both the light and dark beds that constitute the cycles are moderately to strongly bioturbated with many recognizable trace fossils such as Planolites, Zoophycos, Chondrites, Teichichnus, and Heliminthoida. Teichichnus and Heliminthoida are relatively rare and the latter is found only above 603.5 mbsf. The burrows are predominantly horizontal. However, downward burrowing mixed lighter sediments locally into the darker underlying beds. Section $122-762 \mathrm{C}-56 \mathrm{X}-2$ contains a typical example (Fig. 4). These burrows indicate that the color variation is primarily a depositional product instead of a diagenetic outcome. Inoceramus fragments are more common in light beds.

\section{Calcium Carbonate and Total Organic Carbon Content}

Calcium carbonate measurements (see Appendix for the details of the method) were carried out on 186 samples from Cores $122-762 \mathrm{C}-47 \mathrm{X}$ through $-56 \mathrm{X}$ (Table 1). The 92 calcium carbonate measurements from light beds vary from $74.71 \%$ to $93.72 \%$ with an average of $86.99 \%$ and a standard deviation of $3.17 \%$. The 94 measurements from dark beds vary from $57.22 \%$ to $91.5 \%$ with an average of $74.46 \%$ and a standard deviation of $7.03 \%$. Therefore, on average, the calcium carbonate content is about $13 \%$ lower and more variable in the dark beds than in the light beds. Detailed measurements in Cores $122-762 \mathrm{C}-48 \mathrm{X},-49 \mathrm{X},-51 \mathrm{X}$, and $-52 \mathrm{X}$ show that calcium carbonate content of light beds is always higher than that of the nearby dark beds (Fig. 5A), and the calcium carbonate content is more variable in the dark beds than in the light beds.

Total organic carbon (TOC) content is low in both light and dark beds. There is no significant difference in TOC between dark and light beds. The TOC values vary from $0.0 \%$ to $0.05 \%$ in eight dark beds and from 0.0 to $0.02 \%$ in four light beds (Table 2).

\section{Other Compositional Differences}

Whole-rock X-ray diffraction (XRD) analysis (refer to Wilkens, this volume, for details of the method) was carried out, using the same samples for which we have coulometrically determined calcium carbonate content, in Cores 122$762 \mathrm{C}-47 \mathrm{X}$ through $-53 \mathrm{X}$ (Table 1). Only quartz and calcite consistently show reliable peaks on the X-ray spectra. Peaks of clay minerals and other accessory minerals such as feldspar, zeolite, and pyrite were identified when possible. Content of total clay minerals was calculated, and with rare exception, indicated that more clay minerals were present in dark beds than in the adjacent light beds (Fig. 5B). The same tendency is found in the variation of quartz content (Fig. 5C). We also observed that (1) more calcium carbonate occurs in 


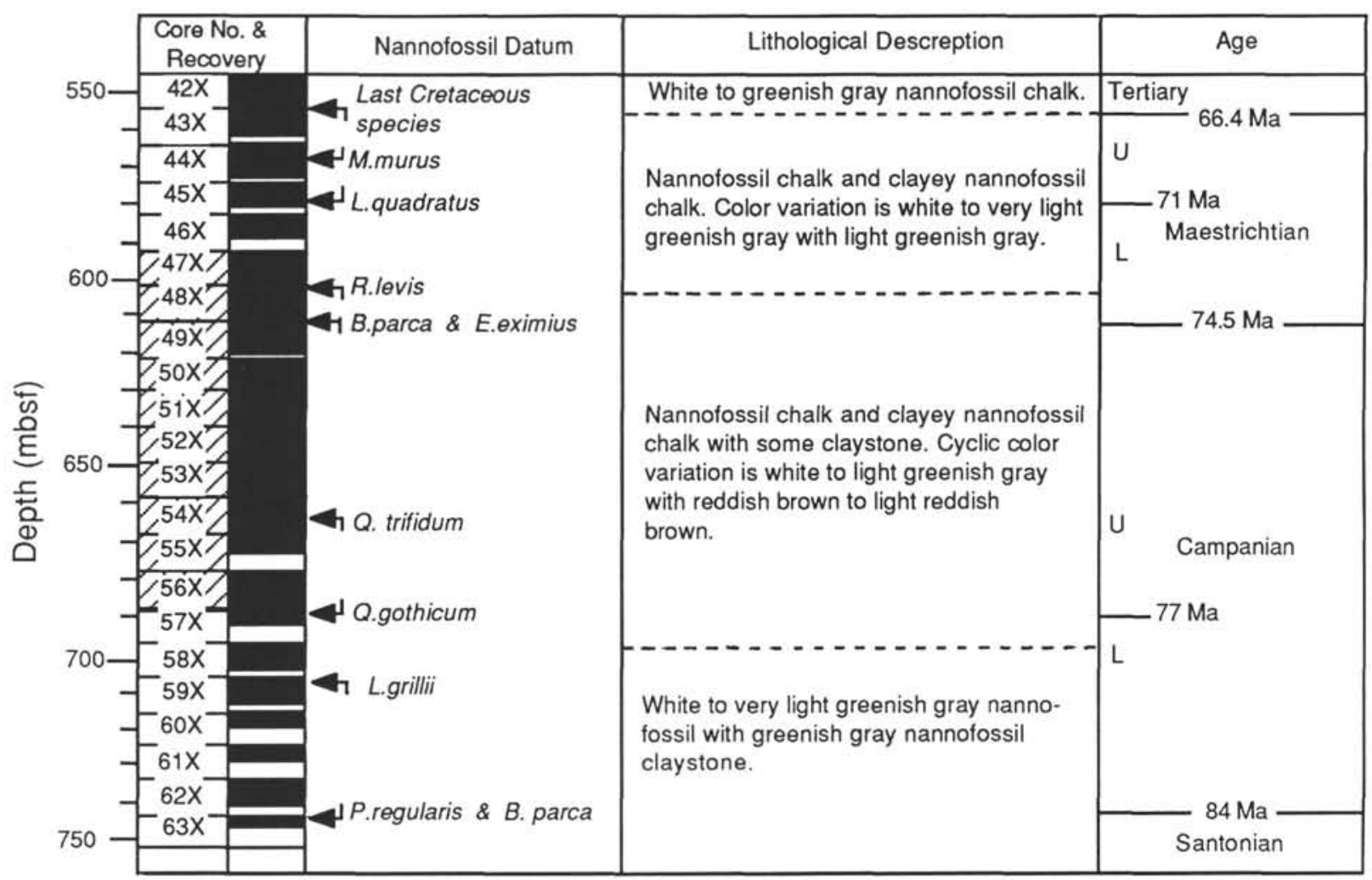

Figure 3. Lithologic and stratigraphic summary of the Campanian to Maestrichtian cyclic sediments in Hole 762C, showing core number, core recovery (in black), nannofossil datum, lithology, and age (Haq, von Rad, O'Connell, et al., 1990). Shaded area indicates the interval of well-developed cyclic color variations. The numeric ages at the stage boundaries are from the time scale by Kent and Gradstein (1985).

light beds than in dark beds, as is supported by the coulometric measurements, (2) more kaolinite and illite peaks than smectite peaks appear in dark beds, but more smectite peaks than kaolinite and illite peaks appear in light beds, and (3) more zeolite and feldspar peaks are detected in dark beds.

Compositional differences between the light and dark beds were also detected in the shipboard smear slide examinations (Table 3 ) of Cores 122-762C-47X through -56X. The differences presented by the smear slide record can be outlined as follows: (1) quartz, clay, and opaques are more common in dark beds than in light beds, (2) zeolites and dolomite are more common in dark beds than in light beds, (3) sponge spicules and ostracodes are more abundant in dark beds than in light beds, (4) biogenic carbon grains and bioclasts are more common in dark beds than in light beds, and (5) nannofossils and foraminifers are more abundant in light beds than in dark beds.

Until now, the source of micropaleontological information for the Upper Cretaceous cyclic sequences from the Exmouth Plateau has been limited to smear slide examination. More work is needed to have a systematic micropaleontological comparison between the dark and light beds. The smear slide observation that there are more nannofossils in the light beds is in agreement with what has been observed in similar cyclic sequences (i.e., Atlantic Lower Cretaceous cyclic sequences, Ogg et al., 1987; and Lower Cretaceous cyclic sequences from Vocontian Basin, Darmedru et al., 1982). This observation can be explained by higher bioproductivity in the surface water during the phase in which the light beds were deposited. However, the smear slide observation that there are more foraminifers in the light beds is not in accordance with other similar cyclic sequences.

\section{Diagenetic Overprint}

The sediments recovered in the studied interval are relatively soft. Diagenetic alteration appears as small, thin seams cutting trace fossils. These seams occur irregularly and more often in dark beds than in light beds. These seams cut trace fossils and result from early diagenetic compaction, local calcium carbonate dissolution, and reprecipitation during burial (Fig. 6). Scanning electron microscope (SEM) examination on seven samples from three and a half cycles in Sections 122-762C-49X-2 through -49X-3 exposes etched nannofossils and some secondary deposited calcium carbonate in samples from dark beds and fewer dissolved nannofossils also with some secondary deposited calcium carbonate in samples from light beds (Fig. 7). It is observed that the light beds are more porous and therefore they cannot have a large volume of cement. The effects of the diagenetic process are not profound enough to alter the overall cyclic color alternation produced by depositional processes, as redeposition of calcium carbonate is not restricted to the light beds. Scattered pyrite crystals and a burrow filled by pyrite crystals are found only above 603.5 mbsf.

Physical properties measurements (Table 4) show that light beds have higher water content, lower bulk density, slightly lower grain density, and higher porosity than dark beds. This table shows that for both the light and dark beds porosity tends to decrease with increasing depth. As the cyclic sequences in the Indian Ocean are not fully indurated, the influence from cementation is small and the dissimilarity in physical properties (e.g., porosity) may be more directly related to and reflect the differences in compaction between the light and dark beds, caused by the original differences in composition. It is still feasible, however, to quantify the 


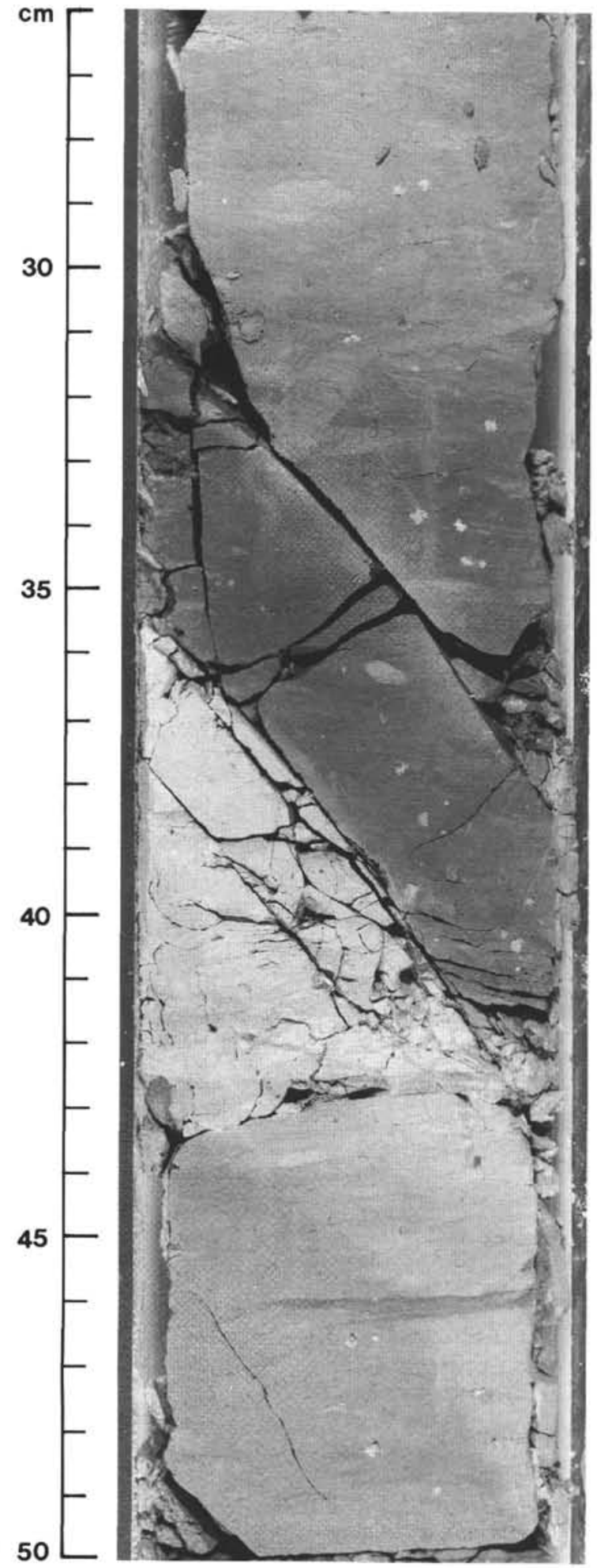

Figure 4. Typical bioturbation that introduces the light sediments from the upper light bed to the lower dark bed. The lower, sharply inclined boundary represents a microfault plane. Sample 122-762C-56X-3, $26-50 \mathrm{~cm}$. difference in compaction. If comparing the mean values of water content and porosity between the light and dark beds, the difference is small, both less than $4.5 \%$. Supposing the original porosity of the light and dark sediments is nearly the same, according to a mean difference of $4.03 \%$ in present porosity between these two sediment, the difference in compaction should also be small. If the original porosity of the light bed is higher than the dark beds, the actual difference in compaction may be smaller; otherwise there might be a greater difference in compaction. In any case, we expect that the influence of differential compaction, if there is any, on statistical analysis of Indian Ocean cyclic sediments would be much smaller than that on older and more indurated cyclic sequences.

\section{Implications}

A summary of the differences between the light and dark beds is given in Table 5. The geological implications from sedimentological examination of the cyclic interval and a comparison of the light and dark beds can be summarized as follows:

The cyclic color alternation in the sediments is a depositional feature and not a diagenetic product. A primary origin for color cyclicity is supported by (1) burrows that introduce the light-colored sediment of an overlying bed into a darkcolored underlying bed and (2) consistently higher calcium carbonate content in light beds.

Strong to moderate bioturbation throughout the cyclic interval and the generally low TOC value in both light and dark beds indicate that the bottom water had sufficient oxygen supply for benthic organisms to survive. The occasionally faint lamination in dark beds may imply bottom water with slightly less oxygen supply and hence the reduction of benthic organisms. The changes in oxygen supply to the bottom water are subtle and are not crucial to the formation of the cycles. Compositional differences between the light beds and dark beds imply that the change lies mainly in sediment supply. When the light beds were deposited, biogenic material was the major component, with less terrestrial input. When the dark beds were deposited, terrestrial input was increased, with some decrease in biogenic supply. The smaller standard deviation of bed thickness and less-variable calcium carbonate content of light beds may suggest that the amount of biogenic supply is less variable than the amount of terrestrial supply. More variability in terrestrial supply indicates that landbased changes may have played a more important role in cyclic sedimentation.

Diagenetic processes may have enhanced the color cyclicity, producing small seams that locally cut trace fossils and local calcium carbonate dissolution/reprecipitation.

A background change in the cyclic sequence is indicated by the overall color change from a brownish-hued interval below 603.5 mbsf to a greenish-hued interval with pyrite crystals above that depth. This background change is also reflected by exclusive occurrence of the trace fossil Heliminthoida above this depth. The background change is believed to have been caused by a relative sea-level change (Haq, von Rad, O'Connell, et al., 1990) from highstand to lowstand. Whatever the cause, the background change does not seem to have affected the formation of the cycles and it is not a controlling factor.

\section{QUANTITATIVE SEARCH FOR PERIODICITY OF CYCLIC SEDIMENTS}

\section{Method and Time Series Generation}

As it is difficult to estimate the regularity of the color cycles visually, a statistical method should be utilized for this purpose. Spectral analysis is frequently employed in the studies of Quaternary cyclic sediments. However, the conventional Fourier spectral analysis method is best suitable for smooth time series 
Table 1. Calcium carbonate, clay, and quartz contents in Cores 122-762C-47X through 122-762C-56X.

\begin{tabular}{|c|c|c|c|c|c|c|c|}
\hline $\begin{array}{l}\text { Core, section, } \\
\text { interval }(\mathrm{cm})\end{array}$ & $\begin{array}{l}\text { Depth } \\
\text { (mbsf) }\end{array}$ & $\begin{array}{r}\mathrm{CaCO}_{3} \\
(\%)^{\mathrm{a}^{2}}\end{array}$ & $\begin{array}{l}\mathrm{CaCO}_{3} \\
(\%)^{\mathrm{b}^{3}}\end{array}$ & $\begin{array}{l}\text { Clay } \\
(\%)^{b}\end{array}$ & $\begin{array}{l}\text { Quartz } \\
\text { peaks }\end{array}$ & Mineral $^{c}$ & Color \\
\hline \multicolumn{8}{|l|}{$122-762 \mathrm{C}$. } \\
\hline $47 X-1,9-11$ & 592.59 & 82.81 & 87.3 & 9.3 & 3.3 & $\mathrm{~K}, \mathrm{I}, \mathrm{S}$ & dark (g) \\
\hline $47 X-1,95-97$ & 593.35 & 91.69 & 90.3 & 8.5 & 1.2 & $\mathrm{~K}, \mathrm{~S}, \mathrm{I}$ & light (g) \\
\hline$d_{47 X-2,81-83}$ & 594.81 & 82.74 & & & & & dark (g) \\
\hline $47 X-2,128-130$ & 595.28 & 88.82 & 89.4 & 8.1 & 2.5 & $\mathrm{~S}$ & light (g) \\
\hline$d_{47 X-2,140-142}$ & 595.40 & 71.99 & & & & & dark (g) \\
\hline $47 X-3,15-17$ & 595.65 & 78.80 & 82.9 & 10.7 & 6.5 & $\mathrm{~K}, \mathrm{I}, \mathrm{S}$ & dark (g) \\
\hline $47 X-3,40-42$ & 595.90 & 86.90 & 85.3 & 10.8 & 3.9 & $\mathrm{~K}$ & light (g) \\
\hline $47 X-4,40-42$ & 597.40 & 86.79 & 91.4 & 6.2 & 2.4 & 1 & light $(\mathrm{g})$ \\
\hline$d_{47 X-4,71-73}$ & 597.71 & 84.33 & & & & & dark (g) \\
\hline$d_{47 X-4,106-108}$ & 598.06 & $\begin{array}{l}84.33 \\
87.41\end{array}$ & & & & & light (g) \\
\hline $47 X-5,23-25$ & 598.73 & 73.43 & 77.4 & 13.8 & 8.8 & $\mathrm{~K}, \mathrm{I}, \mathrm{S}$ & dark (g) \\
\hline $47 X-5,59-60$ & 599.09 & 88.65 & 95.2 & 2.5 & 2.3 & $\mathrm{~K}, \mathrm{~S}$ & light (g) \\
\hline $47 X-6,23-25$ & 600.23 & 66.36 & & & & & dark (g) \\
\hline $\mathrm{d}_{47 \mathrm{X}-6,66-68}$ & 600.66 & 82.74 & & & & & dark (g) \\
\hline $47 X-6,85-86$ & 600.83 & 88.92 & 95.0 & 2.9 & 2.1 & S & light (g) \\
\hline $48 X-1,4-5$ & 602.04 & 81.48 & 83.1 & 11 & 5.9 & S & dark (g) \\
\hline $48 X-1,40-42$ & 602.40 & 85.78 & 93.0 & 4.2 & 2.8 & K, S & light $(\mathrm{g})$ \\
\hline$d_{48 X-1,90-92}$ & 602.90 & 88.41 & & & & & light (g) \\
\hline $48 X-1,100-101$ & 603.00 & 80.26 & 83.5 & 11.5 & 5.0 & $\mathrm{~K}, \mathrm{~S}$ & dark (g) \\
\hline $48 X-1,138-139$ & 603.38 & 88.83 & 88.0 & 9.7 & 2.4 & $\mathrm{~K}$ & light (g) \\
\hline $48 X-2,19-20$ & 603.69 & 85.02 & 86.4 & 9.9 & 3.7 & 1 & light (b) \\
\hline $48 X-2,35-36$ & 603.85 & 80.85 & 81.9 & 11.5 & 6.6 & K & dark (b) \\
\hline $48 X-2,45-47$ & 603.95 & 80.83 & 83.3 & 11.9 & 4.8 & $\mathrm{~K}, \mathrm{~S}$ & light (b) \\
\hline $48 X-2,61-61$ & 604.11 & 67.77 & 72.4 & 19.0 & 8.6 & $\mathrm{~K}, \mathrm{~S}$ & dark (b) \\
\hline $48 X-2,71-72$ & 604.21 & 85.52 & 87.7 & 8.6 & 3.7 & $\mathrm{~K}, \mathrm{I}, \mathrm{S}$ & light (b) \\
\hline $48 X-2,80-81$ & 604.30 & 65.26 & 75.5 & 14.7 & 9.8 & $\mathrm{~K}, \mathrm{~S}, \mathrm{~F}$ & dark (b) \\
\hline $48 X-2,93-94$ & 604.43 & 84.10 & 86.3 & 8.6 & 5.1 & $\mathrm{~K}, \mathrm{~S}, \mathrm{I}$ & light (b) \\
\hline $48 X-2,105-106$ & 604.55 & 79.11 & 85.9 & 6.9 & 7.2 & $\mathrm{~K}, \mathrm{~S}$ & dark (b) \\
\hline $48 X-2,114-115$ & 604.64 & 85.76 & 85.4 & 10.9 & 3.7 & $\mathrm{~K}, \mathrm{I}, \mathrm{F}$ & light (b) \\
\hline $48 X-2,132-133$ & 604.82 & 68.28 & 70.2 & 16.7 & 13.1 & $\mathrm{~K}, \mathrm{I}, \mathrm{S}$ & dark (b) \\
\hline $48 X-3,20-21$ & 605.20 & 85.71 & 86.6 & 9.8 & 3.6 & $\mathrm{~K}, \mathrm{~S}$ & light (b) \\
\hline $48 X-3,57-58$ & 605.57 & 78.92 & 81.5 & 12.4 & 6.1 & $\mathrm{~K}$ & dark (b) \\
\hline $48 X-3,74-75$ & 605.74 & 88.48 & 87.9 & 9.4 & 2.7 & $\mathrm{~S}$ & light (b) \\
\hline$d_{48 X-3,94-96}$ & 605.94 & 84.16 & & & & & dark (b) \\
\hline $48 X-3,129-130$ & 606.29 & 87.12 & 90.2 & 6.7 & 3.1 & $\mathrm{~K}, \mathrm{~S}$ & light (b) \\
\hline $48 X-4,10-11$ & 606.60 & 79.50 & 87.6 & 7.2 & 5.2 & $\mathrm{~K}, \mathrm{~S}$ & dark (b) \\
\hline $48 \times-4,17-18$ & 606.67 & 85.03 & 84.0 & 9.6 & 6.4 & $\mathrm{~K}, \mathrm{~S}$ & light (b) \\
\hline $48 X-4,30-31$ & 606.80 & 61.36 & 69.5 & 16.8 & 13.8 & $\mathrm{~K}, \mathrm{I}, \mathrm{S}, \mathrm{F}$ & dark (b) \\
\hline $48 X-4,39-40$ & 606.89 & 83.82 & 86.0 & 9.3 & 4.7 & K, I, S & light (b) \\
\hline $48 X-4,54-55$ & 607.04 & 75.11 & 80.9 & 11.6 & 7.4 & $\mathrm{~K}, \mathrm{l}, \mathrm{S}, \mathrm{F}$ & dark (b) \\
\hline $48 X-4,73-75$ & 607.23 & 85.50 & 88.2 & 7.9 & 3.9 & $\mathrm{~S}$ & light (b) \\
\hline $48 X-4,85-86$ & 607.35 & 73.47 & 76.6 & 15.7 & 7.7 & K, I & dark (b) \\
\hline $48 X-4,103-105$ & 607.53 & 84.07 & 85.3 & 10.7 & 4.0 & $\mathrm{~S}, \mathrm{~K}, \mathrm{Z}$ ? & light (b) \\
\hline $48 X-4,120-121$ & 607.70 & 76.91 & 76.4 & 14.3 & 9.3 & $\mathrm{~K}, \mathrm{l}, \mathrm{S}$ & dark (b) \\
\hline $48 X-4,138-140$ & 607.88 & 86.68 & 93.2 & 1.4 & 5.4 & $\mathrm{~K}, \mathrm{l}, \mathrm{S}$ & light (b) \\
\hline$d_{48 X}-5,55-57$ & 608.55 & 76.33 & 77.6 & 15.0 & 7.0 & $\mathrm{~K}, \mathrm{~S}, \mathrm{Z}, \mathrm{F}$ & dark (b) \\
\hline $48 X-5,78-80$ & 608.78 & 91.30 & 91.6 & 6.7 & 1.7 & & light (b) \\
\hline $48 X-5,111-113$ & 609.11 & 62.61 & 68.2 & 17.2 & 14.6 & $\mathrm{~K}, \mathrm{I}$ & dark (b) \\
\hline $48 X-5,122-124$ & 609.22 & 85.67 & 87.4 & 8.5 & 4.1 & & light (b) \\
\hline $48 X-5,145-146$ & 609.45 & 84.17 & 83.6 & 12.0 & 4.5 & $\mathrm{~K}$ & dark (b) \\
\hline$d_{48 X}-6,68-70$ & 610.18 & 89.41 & & & & & light (b) \\
\hline $48 X-6,87-89$ & 610.37 & 61.88 & 65.9 & 25.0 & 9.0 & $\mathrm{~K}, \mathrm{~S}$ & dark (b) \\
\hline $48 X-6,97-99$ & 610.47 & 79.88 & 75.9 & 12.3 & 11.8 & $S, 1$ & light (b) \\
\hline $48 X-6,104-106$ & 610.54 & 85.32 & 87.0 & 8.2 & 4.8 & $\mathrm{~K}$ & light (b) \\
\hline $48 X-6,117-118$ & 610.67 & 89.15 & 90.9 & 5.4 & 3.6 & & light (b) \\
\hline $48 X-6,147-149$ & 610.97 & 79.05 & 83.5 & 8.5 & 8.0 & $\mathrm{~K}, \mathrm{I}$ & dark (b) \\
\hline $49 X-1,7-9$ & 611.57 & 74.71 & 88.1 & 8.7 & 3.2 & & light (b) \\
\hline$d_{49 X-1,9-11}$ & 611.59 & 90.41 & & & & & light (b) \\
\hline $49 \mathrm{X}-1,23-25$ & 611.75 & 63.61 & 73.3 & 13.9 & 12.8 & $\mathrm{~K}, \mathrm{I}, \mathrm{S}$ & dark (b) \\
\hline $49 X-1,61-62$ & 612.11 & 91.92 & 89.3 & 9.2 & 1.5 & $\mathrm{~S}, \mathrm{~K}, \mathrm{Z}$ ? & light (b) \\
\hline $49 X-1,95-96$ & 612.45 & 85.80 & 86.4 & 8.6 & 5.0 & $\mathrm{~K}$ & dark (b) \\
\hline $49 X-1,116-117$ & 612.66 & 89.56 & 91.6 & 5.6 & 2.8 & & light (b) \\
\hline $49 X-2,20-21$ & 613.20 & 75.14 & 78.9 & 11.5 & 9.5 & K, I, S & dark (b) \\
\hline $49 X-2,82-83$ & 613.82 & 84.30 & 81.1 & 12.2 & 6.7 & $\mathrm{~K}, \mathrm{I}$ & light (b) \\
\hline $49 X-2,100-101$ & 614.00 & 70.19 & 74.2 & 12.3 & 13.5 & $\mathrm{~K}, \mathrm{I}, \mathrm{S}$ & dark (b) \\
\hline $49 X-2,112-113$ & 614.12 & 84.40 & 82.6 & 10.4 & 7.0 & $\begin{array}{l}n \\
S\end{array}$ & light (b) \\
\hline $49 X-3,20-21$ & 614.70 & 73.93 & 75.1 & 14.0 & 10.9 & $\mathrm{~K}, \mathrm{I}, \mathrm{S}$ & dark (b) \\
\hline $49 X-3,38-39$ & 614.88 & 82.40 & 83.7 & 10.6 & 5.7 & 1 & light (b) \\
\hline $49 X-3,55-56$ & 615.05 & 69.96 & 72.1 & 16.4 & 11.5 & $\mathrm{~S}, \mathrm{I}, \mathrm{K}$ & dark (b) \\
\hline $49 X-3,65-67$ & 615.15 & 75.88 & 78.3 & 13.6 & 8.1 & $\mathrm{~K}, \mathrm{~S}$ & light (b) \\
\hline$d_{49 X-3,78-80}$ & 615.28 & 69.83 & & & & & dark (b) \\
\hline $49 \mathrm{X}-3,89-90$ & 615.39 & 84.54 & 87.1 & 7.4 & 5.5 & $\mathrm{~K}, \mathrm{I}, \mathrm{S}$ & light (b) \\
\hline $49 \times-3,105-106$ & 615.55 & 76.44 & 81.4 & 10.1 & 8.5 & $\mathrm{~K}, \mathrm{I}, \mathrm{S}$ & dark (b) \\
\hline $49 X-3,120-121$ & 615.70 & 89.38 & 96.3 & 0.0 & 3.7 & & light (b) \\
\hline $49 X-3,130-131$ & 615.80 & 77.15 & 80.7 & 10.2 & 9.1 & $\mathrm{~K}, \mathrm{I}, \mathrm{S}$ & dark (b) \\
\hline
\end{tabular}

Table 1 (continued).

\begin{tabular}{|c|c|c|c|c|c|c|c|}
\hline $\begin{array}{l}\text { Core, section, } \\
\text { interval (cm) }\end{array}$ & $\begin{array}{l}\text { Depth } \\
\text { (mbsf) }\end{array}$ & $\begin{array}{l}\mathrm{CaCO}_{3} \\
(\%)^{\mathrm{a}^{3}}\end{array}$ & $\begin{array}{l}\mathrm{CaCO}_{3} \\
(\%)^{b^{3}}\end{array}$ & $\begin{array}{l}\text { Clay } \\
(\%)^{b}\end{array}$ & $\begin{array}{l}\text { Quartz } \\
\text { peaks }^{\mathrm{c}}\end{array}$ & Mineral $^{c}$ & Color \\
\hline$d_{49 X-4,19-20}$ & 616.19 & 92.24 & 96.6 & 1.2 & 2.2 & $\mathrm{Z}$ & light (b) \\
\hline $49 X-4,93-94$ & 616.93 & 84.52 & 82.7 & 13.6 & 3.7 & $\mathrm{I}, \mathrm{S}$ & dark (b) \\
\hline $49 \mathrm{X}-4,105-106$ & 617.05 & 90.27 & 91.9 & 5.5 & 2.5 & $\mathrm{~K}, \mathrm{I}$ & light (b) \\
\hline $49 \mathrm{X}-4,117-118$ & 617.17 & 65.41 & 76.5 & 11.9 & 11.6 & $\mathrm{~K}, \mathrm{I}$ & dark (b) \\
\hline $49 \mathrm{X}-4,134-135$ & 617.34 & 89.89 & 91.4 & 6.0 & 2.7 & $\mathrm{I}, \mathrm{S}$ & light (b) \\
\hline $49 \mathrm{X}-4,20-21$ & 617.70 & 87.85 & 87.2 & 8.5 & 4.3 & $\mathrm{~K}$ & dark (b) \\
\hline $49 \times-5,35-36$ & 617.85 & 88.94 & 95.7 & 0.0 & 4.3 & & light (b) \\
\hline $49 \mathrm{X}-5,50-51$ & 618.00 & 72.39 & 76.0 & 14.5 & 9.4 & $\mathrm{~K}, \mathrm{I}, \mathrm{F}$ & dark (b) \\
\hline $49 X-5,80-81$ & 618.30 & 86.10 & 85.0 & 11.0 & 4.0 & $\mathrm{~K}, \mathrm{~S}$ & light (b) \\
\hline $49 \mathrm{X}-5,95-96$ & 618.45 & 78.50 & 81.3 & 7.4 & 11.2 & $\mathrm{~K}, \mathrm{I}$ & dark (b) \\
\hline $49 \mathrm{X}-5,124-125$ & 618.74 & 87.62 & 88.7 & 6.9 & 4.4 & $K, S$ & light (b) \\
\hline $\mathrm{d}_{49} \mathrm{X}-5,132-134$ & 618.82 & 79.24 & & & & & dark (b) \\
\hline $49 \mathrm{X}-5,135-136$ & 618.85 & 81.85 & 74.8 & 18.2 & 7.0 & $\mathrm{~K}, \mathrm{I}$ & dark (b) \\
\hline $50 \mathrm{X}-1,80-81$ & 621.80 & 77.44 & 79.9 & 9.6 & 10.5 & $\mathrm{~K}, \mathrm{I}, \mathrm{S}$ & dark (b) \\
\hline $\mathrm{d}_{50 \mathrm{X}-2,48-50}$ & 622.98 & 81.33 & & & & & dark (b) \\
\hline $50 X-2,95-96$ & 623.45 & 87.42 & 89.3 & 6.3 & 4.4 & & light (b) \\
\hline $50 \mathrm{X}-2,114-115$ & 623.64 & 75.13 & 82.8 & 7.2 & 9.9 & & dark (b) \\
\hline $50 \mathrm{X}-3,45-46$ & 624.45 & 87.05 & 88.5 & 7.3 & 4.2 & $\mathrm{~K}, \mathrm{I}, \mathrm{S}$ & light (b) \\
\hline $50 \mathrm{X}-3,91-92$ & 624.91 & 68.68 & 73.4 & 13.5 & 13.1 & $\mathrm{~K}, \mathrm{I}, \mathrm{S}$ & dark (b) \\
\hline $\mathrm{d}_{50 \mathrm{X}-4,94-96}$ & 624.44 & 87.41 & & & & & light (b) \\
\hline $50 X-4,116-117$ & 626.66 & 77.86 & 80.4 & 9.7 & 9.9 & $1, \mathrm{~S}$ & dark (b) \\
\hline $50 X-5,20-21$ & 627.20 & 74.32 & 79.6 & 11.1 & 9.3 & $\mathrm{~S}, \mathrm{~K}, \mathrm{I}, \mathrm{F}$ & dark (b) \\
\hline $50 X-5,55-56$ & 627.55 & 90.66 & 95.9 & 1.3 & 2.8 & & light (b) \\
\hline $50 \mathrm{X}-6,20-21$ & 628.70 & 90.63 & 89.2 & 7.6 & 3.2 & & light (b) \\
\hline 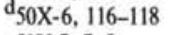 & 629.66 & 85.16 & & & & & light (b) \\
\hline $50 \times-7,7-8$ & 630.07 & 88.60 & 91.1 & 4.7 & 4.2 & & light (b) \\
\hline $50 X-7,20-21$ & 630.20 & 73.48 & 79.8 & 11.1 & 9.1 & $\mathrm{~K}, \mathrm{I}, \mathrm{S}$ & dark (b) \\
\hline $51 \mathrm{X}-1,38-40$ & 630.88 & 74.54 & 79.7 & 11.0 & 9.3 & $\mathrm{~K}, \mathrm{I}$ & dark (b) \\
\hline $51 X-1,114-116$ & 631.64 & 89.60 & 89.9 & 6.6 & 3.5 & & light (b) \\
\hline $51 X-1,140-141$ & 631.90 & 84.23 & 85.6 & 8.0 & 6.4 & S, I & dark (b) \\
\hline $51 X-2,5-6$ & 632.05 & 88.22 & 88.1 & 7.9 & 3.9 & $\mathrm{~S}, \mathrm{~K}, \mathrm{Z}$ ? & light (b) \\
\hline $\mathrm{d}_{51 X-2,66-68}$ & 632.66 & 73.08 & & & & & dark (b) \\
\hline $51 X-2,68-69$ & 632.68 & 72.84 & 74.5 & 16.2 & 9.3 & $\mathrm{I}, \mathrm{S}$ & dark (b) \\
\hline $51 X-2,81-82$ & 632.81 & 78.80 & 81.5 & 10.2 & 8.3 & $\mathrm{~K}, \mathrm{~S}$ & dark (b) \\
\hline $51 \mathrm{X}-2,120-121$ & 633.20 & 75.96 & 80.9 & 9.8 & 9.3 & $\mathrm{~K}, \mathrm{I}, \mathrm{S}$ & dark (b) \\
\hline $51 X-3,50-51$ & 634.00 & 75.74 & 78.5 & 11.7 & 9.9 & $\mathrm{~K}, \mathrm{~S}, \mathrm{Z}$ ? & dark (b) \\
\hline $51 X-3,94-95$ & 634.44 & 86.23 & 83.7 & 10.7 & 5.6 & I & light (b) \\
\hline $51 X-3,109-110$ & 634.59 & 69.58 & 73.9 & 14.5 & 11.6 & $\mathrm{~K}, \mathrm{I}, \mathrm{S}$ & dark (b) \\
\hline $51 \mathrm{X}-3,120-121$ & 634.70 & 90.84 & 90.2 & 7.0 & 2.8 & $\mathrm{~s}$ & light (b) \\
\hline $51 \mathrm{X}-3,139-140$ & 634.89 & 75.53 & 76.6 & 12.1 & 11.3 & $\mathrm{~K}, \mathrm{I}, \mathrm{S}, \mathrm{Z}$ ? & dark (b) \\
\hline $51 X-4,5-6$ & 635.05 & 93.72 & 89.3 & 7.8 & 2.8 & $\mathrm{~S}, \mathrm{Z}$ ? & light (b) \\
\hline $51 X-4,25-26$ & 635.25 & 74.72 & 77.3 & 12.6 & 10.2 & $\mathrm{~K}, \mathrm{~S}$ & dark (b) \\
\hline $51 X-4,45-46$ & 635.45 & 87.32 & 95.8 & 0.0 & 4.2 & & light (b) \\
\hline $51 X-4,60-61$ & 635.60 & 87.15 & 79.9 & 15.6 & 4.7 & & dark (b) \\
\hline $51 X-4,78-80$ & 635.78 & 87.47 & 95.9 & 0.0 & 4.1 & & light (b) \\
\hline $51 X-4,90-91$ & 635.90 & 80.18 & 81.2 & 11.0 & 7.8 & $\mathrm{~K}, \mathrm{I}, \mathrm{S}$ & dark (b) \\
\hline $51 X-4,107-108$ & 636.07 & 86.35 & 86.5 & 7.7 & 5.8 & $\mathrm{~K}, \mathrm{~S}$ & light (b) \\
\hline $51 X-4,125-127$ & 636.25 & 81.70 & 86.0 & 7.4 & 6.6 & $\mathrm{~K}$ & dark (b) \\
\hline$d_{51 X-4,142-144}$ & 636.42 & 85.49 & 83.3 & 11.5 & 5.2 & S, I, K, Z & dark (b) \\
\hline $51 X-4,147-148$ & 636.47 & 87.57 & 93.2 & 11.0 & 5.8 & & light (b) \\
\hline $51 X-5,7-8$ & 636.57 & 87.91 & 86.4 & 9.4 & 4.2 & & light (b) \\
\hline $51 X-5,17-18$ & 636.67 & 84.46 & 87.1 & 8.2 & 4.7 & & dark (b) \\
\hline $51 X-5,70-72$ & 637.20 & 87.13 & 94.6 & 0.0 & 5.4 & & light (b) \\
\hline $51 X-5,120-122$ & 637.70 & 75.72 & 71.7 & 18.1 & 10.1 & $\mathrm{~K}, \mathrm{I}, \mathrm{S}$ & dark (b) \\
\hline $51 X-5,142-144$ & 637.92 & & & & & & dark (b) \\
\hline $52 X-1,49-51$ & 640.49 & 73.74 & 74.8 & 13.6 & 11.7 & $\mathrm{~K}, \mathrm{I}$ & dark (b) \\
\hline $52 X-1,66-69$ & 640.66 & 84.84 & 92.4 & 0.0 & 7.6 & & light (b) \\
\hline $52 X-1,90-91$ & 640.90 & 80.71 & 80.8 & 9.8 & 9.4 & & dark (b) \\
\hline $52 \mathrm{X}-1,100-102$ & 641.00 & 88.17 & 86.8 & 6.4 & 6.8 & & light (b) \\
\hline $52 X-1,115-117$ & 641.15 & 80.06 & 84.4 & 6.6 & 9.0 & & dark (b) \\
\hline $52 \mathrm{X}-1,135-137$ & 641.35 & 93.68 & 87.7 & 5.7 & 6.6 & $\mathrm{~K}, \mathrm{I}$ & light (b) \\
\hline $52 \mathrm{X}-2,49-50$ & 641.99 & 85.32 & 82.7 & 9.8 & 7.5 & $\mathrm{~K}, \mathrm{~S}$ & light (b) \\
\hline$d_{52 X-2,50-52}$ & 642.00 & 85.66 & & & & & light (b) \\
\hline $52 \times-2,77-79$ & 642.27 & 78.56 & 82.4 & 8.3 & 9.4 & & dark (b) \\
\hline $52 X-2,113-115$ & 642.63 & 85.84 & & 8.0 & 5.9 & $\mathrm{~K}$, & light (b) \\
\hline $52 X-2,121-123$ & 642.71 & 82.18 & 83.7 & 7.5 & 8.8 & $\mathrm{~K}, \mathrm{I}$ & dark (b) \\
\hline $52 X-2,139-141$ & 642.89 & 87.22 & 86.4 & 8.9 & 4.7 & $\mathrm{~K}, \mathrm{~S}$ & light (b) \\
\hline $52 \mathrm{X}-3,29-30$ & 643.29 & 77.70 & 77.0 & 13.1 & 9.9 & $\mathrm{~K}$ & dark (b) \\
\hline $52 X-3,44-46$ & 643.44 & 86.77 & 83.6 & 10.4 & 6.0 & $\mathrm{~K}, \mathrm{I}, \mathrm{S}$ & light (b) \\
\hline $52 X-3,77-78$ & 643.77 & 88.59 & 85.0 & 9.7 & 5.4 & & light (b) \\
\hline $52 X-3,103-105$ & 644.03 & 84.03 & 83.3 & 9.0 & 7.6 & $\mathrm{~K}, \mathrm{I}, \mathrm{Z}$ ? & dark (b) \\
\hline $52 X-3,124-126$ & 644.24 & 87.07 & 87.2 & 7.4 & 5.4 & $\mathrm{I}, \mathrm{S}$ & light (b) \\
\hline $52 X-3,140-141$ & 644.40 & 68.97 & 70.9 & 13.4 & 15.7 & $\mathrm{~K}, \mathrm{I}$ & dark (b) \\
\hline $52 X-4,14-16$ & 644.64 & 86.76 & 87.9 & 5.9 & 6.1 & $\mathrm{~s}$ & light (b) \\
\hline $52 X-4,71-73$ & 645.21 & 81.44 & 80.3 & 10.5 & 9.1 & $\mathrm{~K}, \mathrm{l}, \mathrm{S}$ & \\
\hline $\mathrm{d}_{52 \mathrm{X}-4,76-78}$ & 645.26 & 81.91 & & & & s & dark (b) \\
\hline $52 \mathrm{X}-4,111-113$ & 645.61 & 88.47 & 87.5 & 7.8 & 6.4 & S & light (b) \\
\hline & & & & & & & \\
\hline
\end{tabular}


Table 1 (continued).

\begin{tabular}{|c|c|c|c|c|c|c|c|}
\hline $\begin{array}{l}\text { Core, section, } \\
\text { interval }(\mathrm{cm})\end{array}$ & $\begin{array}{l}\text { Depth } \\
\text { (mbsf) }\end{array}$ & $\begin{array}{l}\mathrm{CaCO}_{3} \\
(\%)^{\mathrm{a}}\end{array}$ & $\begin{array}{l}\mathrm{CaCO}_{3} \\
(\%)^{b^{3}}\end{array}$ & $\begin{array}{l}\text { Clay } \\
(\%)^{b}\end{array}$ & $\begin{array}{l}\text { Quartz } \\
\text { peaks }\end{array}$ & Mineral $^{c}$ & Color \\
\hline $52 X-5,70-72$ & 646.70 & 81.43 & 86.0 & 5.3 & 8.7 & $\mathrm{~K}, \mathrm{I}$ & dark (b) \\
\hline $52 X-5,132-134$ & 647.32 & 86.95 & 87.4 & 8.1 & 4.5 & $\mathrm{~S}, \mathrm{~K}$ & light (b) \\
\hline $52 X-6,14-16$ & 647.64 & 80.48 & 82.1 & 9.3 & 8.6 & $\mathrm{~K}, \mathrm{I}$ & dark (b) \\
\hline $52 X-6,29-30$ & 647.79 & 88.39 & 84.3 & 10.8 & 4.9 & $\mathrm{~K}$ & light (b) \\
\hline $52 \mathrm{X}-6,48-50$ & 647.98 & 77.64 & 76.2 & 12.9 & 10.8 & $\mathrm{~K}, \mathrm{I}, \mathrm{S}$ & dark (b) \\
\hline $52 X-6,69-71$ & 648.19 & 88.56 & 84.3 & 9.6 & 6.2 & $\mathrm{~S}, 1$ & light (b) \\
\hline $52 X-6,104-106$ & 648.54 & 62.56 & 68.4 & 13.0 & 18.6 & $\mathrm{~K}, \mathrm{I}, \mathrm{S}$ & dark (b) \\
\hline $52 \times-7,29-30$ & 649.29 & 79.78 & 79.3 & 13.0 & 7.7 & $\mathrm{~K}, \mathrm{I}, \mathrm{S}$ & light (b) \\
\hline $53 \mathrm{X}-1,109-110$ & 650.59 & 57.22 & 64.5 & 15.7 & 19.7 & $\mathrm{~K}, \mathrm{I}, \mathrm{S}$ & dark (b) \\
\hline $\mathrm{d}_{53 \mathrm{X}-2,20-22}$ & 651.20 & 84.41 & & & & & dark (b) \\
\hline $53 X-2,90-92$ & 651.90 & 88.52 & 91.5 & 2.2 & 6.3 & $\mathrm{~K}$ & light (b) \\
\hline $53 \times-2,134-135$ & 652.34 & 68.31 & 71.6 & 14.3 & 14.1 & $\mathrm{~K}, \mathrm{I}, \mathrm{S}$ & dark (b) \\
\hline $53 \mathrm{X}-3,10-12$ & 652.60 & 85.87 & 90.4 & 1.9 & 7.7 & $\mathrm{~K}$ & light (b) \\
\hline $53 \mathrm{X}-3,33-35$ & 652.83 & 64.70 & 67.5 & 19.1 & 13.3 & $\mathrm{~K}, \mathrm{I}$ & dark (b) \\
\hline $53 \mathrm{X}-3,99-100$ & 653.49 & 86.26 & 84.9 & 7.1 & 8.1 & $\mathrm{~K}, \mathrm{I}, \mathrm{S}$ & light (b) \\
\hline $53 X-4,89-90$ & 654.89 & 78.16 & 77.5 & 10.9 & 11.5 & $\mathrm{~K}$ & light (b) \\
\hline$d_{53 X}-4,94-97$ & 654.94 & 81.49 & & & & & light (b) \\
\hline $53 X-4,108-110$ & 655.08 & 91.44 & 86.2 & 7.4 & 6.4 & $\mathrm{~K}, \mathrm{~S}$ & light (b) \\
\hline $53 X-5,110-111$ & 656.60 & 69.53 & 73.9 & 9.6 & 16.6 & $\mathrm{~K}, \mathrm{I}, \mathrm{S}$ & dark (b) \\
\hline$d_{53 X}-6,8-10$ & 657.08 & 88.66 & & & & & light (b) \\
\hline $53 \mathrm{X}-6,10-11$ & 657.10 & 87.57 & 86.4 & 8.5 & 5.2 & & light (b) \\
\hline $53 \mathrm{X}-6,30-32$ & 657.30 & 78.96 & 79.9 & 10.4 & 9.7 & $\mathrm{~K}, \mathrm{~S}$ & dark (b) \\
\hline $53 \times-6,74-76$ & 657.74 & 88.15 & 86.6 & 8.7 & 4.7 & $\mathrm{~K}, \mathrm{~S}$ & light (b) \\
\hline $53 \mathrm{X}-6,124-126$ & 658.24 & 79.35 & 79.5 & 12.0 & 8.6 & $\mathrm{~K}$ & dark (b) \\
\hline${ }_{54 X-2,31-33}$ & 660.81 & 87.24 & & & & & light (b) \\
\hline$d_{54 X-4,49-52}$ & 663.99 & 80.83 & & & & & dark (b) \\
\hline $\mathrm{d}_{54 \mathrm{X}-4,86-88}$ & 664.36 & 76.74 & & & & & dark (b) \\
\hline $\mathrm{d}_{54 \mathrm{X}-6,104-106}$ & 667.54 & 84.66 & & & & & light (b) \\
\hline$d_{55 X-2,67-69}$ & 670.67 & 88.49 & & & & & light (b) \\
\hline$d_{55 X-4,7-9}$ & 673.07 & 85.33 & & & & & dark (b) \\
\hline${ }_{566 X-1,63-65}$ & 678.63 & 85.33 & & & & & dark (b) \\
\hline${ }_{56 X-3,90-92}$ & 681.90 & 84.41 & & & & & dark (b) \\
\hline${ }^{d} 56 x-5,56-58$ & 684.56 & 85.41 & & & & & light (b) \\
\hline
\end{tabular}

Note: Color abbreviations are $(\mathrm{g})=$ greenish hue with scattered pyrite crystals and $(b)=$ brownish hue.

${ }^{a}$ From coulometric method.

b From XRD analysis.

${ }^{\mathrm{c}}$ Identified on XRD spectra. $\mathrm{K}=$ kaolinite, $\mathrm{I}=$ illite, $\mathrm{S}=$ smectite, $\mathrm{Z}=$ zeolite, and $\mathrm{f}=$ feldspar.

d Shipboard measurements (Haq, von Rad, O'Connell, et al., 1990).

such as geophysical logging data or curves built by a large number of closely spaced laboratory-derived data points, such as oxygen/carbon isotopes, calcium carbonate content, and other geochemical measurements. The geophysical logging tool of higher vertical resolution used by ODP is the natural gamma-ray intensity $\log (0.3-\mathrm{m}$ vertical resolution) (Schlumberger, 1987). It would be unable to resolve color alternations thinner than $30 \mathrm{~cm}$. Although we take one sample from every bed for calcium carbonate measurements in some of the cores, the number of data points is still too sparse to build a smooth time series. In this situation it is better to build step-like (discrete) time series on the basis of core inspection and measurement of bed thickness, and apply the Walsh spectral analysis method to the discrete time series (Schwarzacher, 1987). As with Fourier analysis, any time series can be resolved into Walsh functions with different sequences, zero crossings of the time base, an equivalence of frequency in Fourier spectral analysis (Weedon, 1986). Walsh functions are discontinuous waves that take only two values, +1 or -1 . Therefore, Walsh spectral analysis is well suited for analyzing step-like time series (for more details on the method, refer to Beauchamp, 1975). Walsh spectral analysis was applied to geoscience for studying the cyclicity of magnetic reversals (Negi and Tiwari, 1983). Later Weedon (1986) and Schwarzacher (1987) introduced this technique to study cyclic sediments in Europe. One advantage of this method is the speed in transform computation, as only addition and subtraction is required.
The application of spectral analysis to geological problems involves four aspects: time series generation, transform operation, spectrum generation, and interpretation. In our time series generation for Site 762 sediments, we code the light beds with +1 and the dark beds with -1 . Figure 8 is an example of the step-like time series we generated. Time series generation in this way is efficient. However, it is no more than an approximation of the cycles, because in reality the boundary between light and dark beds is gradual with a transitional zone varying from 2 to $12 \mathrm{~cm}$, a graduation which may also be reflected in calcium carbonate content. For the purposes of this study we assume that a single bed was produced under relatively constant depositional conditions. To generate the step-like time series, we placed the color boundary in the middle of the transition zone. Therefore, this approximation can represent the cyclic changes in the sediments. It is the best choice for an interval without a sufficient number of laboratory measurements but with a good lithological record. An advantage of building time series in this way is that it will not introduce a superimposed trend, such as a gradual decrease or increase of a certain parameter (e.g., calcium carbonate content) over the interval.

High recovery in all of the cores in the interval, except Core 122-762C-55X (5 m long), aids our time series generation. Other factors, however, prevent time series analysis of every core. Microfault planes were observed in Sections 122-762C-47X-2 and -56X-3 (Fig. 4). The microfault planes, which offset the cyclic succession, can distort the time series and this distortion is difficult to correct. Whole-round samples are taken in Sections 122-762C-47X-5 (115-140 cm, 140-150 $\mathrm{cm})$ and $122-762 \mathrm{C}-53 \mathrm{X}-4(115-140 \mathrm{~cm}, 140-150 \mathrm{~cm})$. Core $122-762 \mathrm{C}-54 \mathrm{X}$ is not only more expanded ( $>100 \%$ recovery), but also more disturbed than the other cores. For these reasons, Sections $122-762 \mathrm{C}-47 \mathrm{X}-1$ through $-47 \mathrm{X}-5$ and Cores $122-762 \mathrm{C}-53 \mathrm{X}$ through $-56 \mathrm{X}$ were rejected for time series generation. The three time series we have built in the cyclic interval are:

1. Sections $122-762 \mathrm{C}-47 \mathrm{X}-6$ through $-49 \mathrm{X}-\mathrm{CC}(600.0$ 621.0 mbsf);

2. Sections $122-762$ C-50X-1 through $-50 X-6(621.0-629.74$ mbsf);

3. Sections $122-762 \mathrm{C}-51 \mathrm{X}-1$ through $-52 \mathrm{X}-\mathrm{CC}(630.5-649.5$ mbsf).

In Section $122-762 \mathrm{C}-50 \mathrm{X}-2$ there is a 10 -cm-long IW whole-round sample, but we interpolated the color of the whole-round sample based on the surrounding color. Two cores were merged in time series 1 and 3 to increase the length of time base for analysis. An 18-m time series is more than 10 times longer than the longest cycle length observed in the cores. Longer time series would improve the accuracy of the position of frequency peaks. With longer time series it is also possible to examine the stability of the frequency peaks by dividing the time series into two subsections and applying spectral analysis on each of them. However, the cost of using longer time series is the introduction of an additional complication caused by unknown changes in sedimentation rate.

\section{The Power Spectra}

In spectral analysis computation, the step-like time series is first sampled with a user-defined uniform interval. The number of data points for a fast Walsh transform must be equal to a power of 2 . In this study the sampling interval varies slightly with the length of every time series, but is less than 1 $\mathrm{cm}$ in order to guarantee sampling the thinnest bed observed in the cores. The program subsequently performed the Walsh 


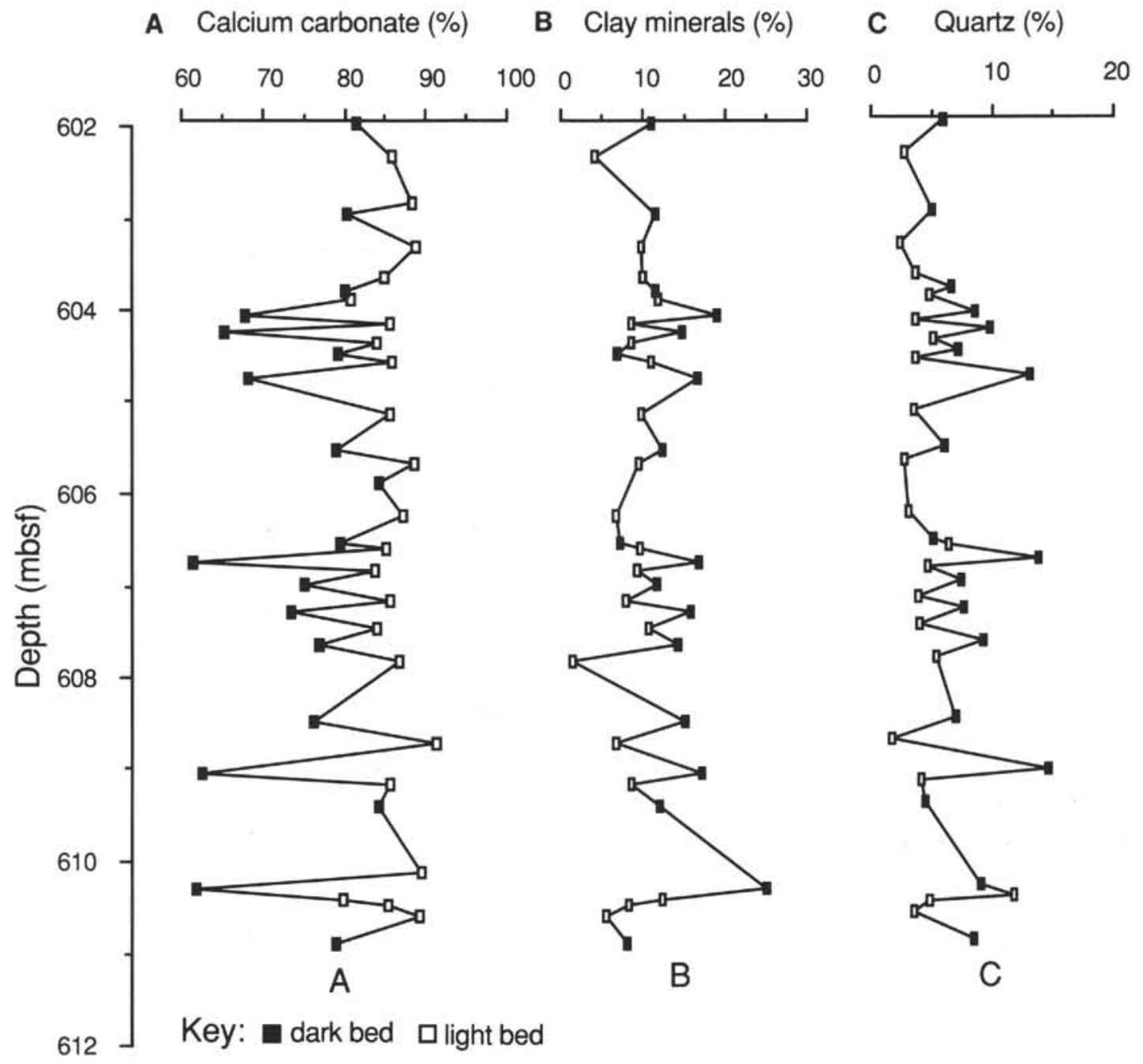

Figure 5. Compositional differences in a succession of cyclic color alternations, Core 122-762C-48X. A. Content of calcium carbonate (\%) measured with coulometric method. The content of calcium carbonate in a light bed is always higher than that of the adjacent dark beds. B. Content of clay minerals (\%) from XRD analysis. There are more clay minerals in dark beds than in light beds, with rare exception. C. Content of quartz (\%) from XRD analysis. Quartz is more abundant in dark beds than in light beds, with rare exception.

transform and power value calculations. The power values were normalized for an application of Fisher's test for white noise (Nowroozi, 1967), and the Walsh spectrum was smoothed using a 3-point Hanning window. The Walsh power spectrum illustrates the relative importance of the frequency peaks. Confidence levels $(90 \%, 95 \%, 98 \%$, and $99 \%)$ were calculated. In this study the $90 \%$ confidence level was used to inspect the validity of the frequency peaks. A component of particular frequency, in terms of zero crossings per meter (cycles $/ \mathrm{m})$, with greater power value indicates a more important role if it is used to recreate the time series. The frequency can also be converted to wavelength in length $(\mathrm{cm})$, the reciprocal of the frequency, or wavelength in time (k.y.) with estimated average sedimentation rate.

Walsh spectral analysis was carried out on the three time series 1,2 , and 3 . The stability of the frequency peaks in a spectrum was tested when analyzing time series 1 and 3 by dividing a time series into two subsections and calculating subsection spectra from them.

The power spectra calculated from time series 1,2 , and 3 are shown in Figures 9, 10, and 11, respectively. On each spectrum the lengths of the time series are indicated as time base, and the bandwidth by a short horizontal bar. A narrower bandwidth ensures higher resolution. The $90 \%$ confidence level is drawn as a horizontal line on both whole-section and subsection spectra. To help interpretation, the ratios of the wavelength of the significant frequency peaks are also indicated. As the subsection is only one-half the length of the whole section, the peaks in the very low-frequency region of the whole-section spectra are difficult to compare with those in subsection spectra in Figures 9 and 11. Therefore, peaks in the low-frequency region (less than $0.5 \mathrm{cycles} / \mathrm{m}$ ) are not to be interpreted and discussed in this study. We regard a frequency peak in a whole-section spectrum to be stable if a strong counterpart is found in one or both of the subsection spectra. The wavelengths of the stable peaks in the higher frequency region are indicated in the figures. In Figures 9 and 11, the location of frequency peaks in the spectra from the subsections deviates slightly from their corresponding stable peaks. The subsection spectra of time series 3 display more variation. The variations may be attributed to change in sedimentation rate and influence from differential compaction. There is no 
Table 2. Total organic carbon in the light and dark beds in Cores 122-762C-47X through 122762C-56X.

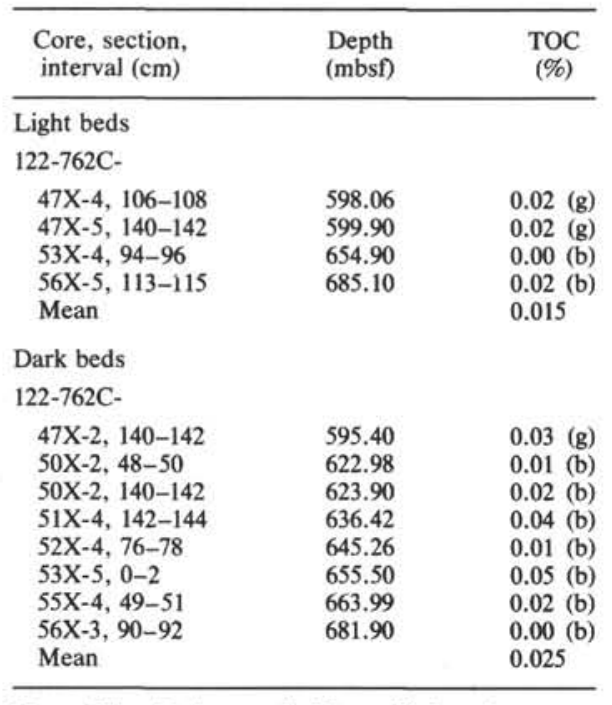

Note: This table is compiled from shipboard measurements (Haq, von Rad, O'Connell, et al., 1990). Color abbreviations are $(\mathrm{g})=$ greenish hue with scattered pyrite crystals and $(b)=$ brownish hue. subsection spectra calculated for time series 2 because of its shorter length. The location of the frequency peaks in Figure 10 is comparable to the location of those appearing in Figures $9 \mathrm{~A}$ and $11 \mathrm{~A}$. Thus, the frequency peaks with wavelengths 84.0 $\mathrm{cm}, 38.2 \mathrm{~cm}$, and $35.0 \mathrm{~cm}$ are interpreted to be stable. The sampling intervals used in analyzing these three time series are less than $1 \mathrm{~cm}$, much smaller than the thickness of the thinnest bed. The Nyquist frequency is at about a 10-k.y. period, judging from the estimated average sedimentation rate $(1.82 \mathrm{~cm} / \mathrm{k} . \mathrm{y}$.). Therefore, the frequency peaks in the spectra are not subjected to an aliasing problem (see Pisias and Mix, 1988). The structure of the spectra does not suggest there is a harmonic problem. We therefore conclude that the stable frequency peaks reveal the predominant frequencies of the color alternations in the cyclic sediments. In Figure 9A, the peak with a $177.8-\mathrm{cm}$ wavelength does not have a counterpart in the spectrum from the upper half of time series 1 (Fig. 9B), but has a counterpart in that from the lower half of the time series wavelength (Fig. 9C), which is longer than $177.8 \mathrm{~cm}$ $(196.6 \mathrm{~cm})$ for the inaccuracy in the lower frequency portion. The peak in Figure 9A with a $40.8-\mathrm{cm}$ wavelength has its counterparts in both subsection spectra (Figs. 9B and 9C), and the peak with a wavelength of $34.3 \mathrm{~cm}$ has a counterpart only in the lower subsection spectrum (Fig. 9C). In Figure 11A, the peaks with $80.1-\mathrm{cm}$ and $70.9-\mathrm{cm}$ wavelengths have only one strong counterpart in the lower subsection spectrum (Fig. $11 \mathrm{C})$. This counterpart is strong and has a shoulder because of

Table 3. Smear slide examination on the composition of the light and dark beds in Cores 122-762C-48X through 122-762C-53X, in percentages.

\begin{tabular}{|c|c|c|c|c|c|c|c|c|c|c|}
\hline $\begin{array}{l}\text { Core, section } \\
\text { interval }(\mathrm{cm})\end{array}$ & Quartz & Feldspar & Clay & $\begin{array}{l}\text { Volcanic } \\
\text { glass }\end{array}$ & $\begin{array}{c}\text { Zeolite + } \\
\text { dolomite }\end{array}$ & $\begin{array}{c}\text { Opaque }+ \\
\text { accessory } \\
\text { minerals }\end{array}$ & Foraminifers & Nannofossils & $\begin{array}{l}\text { Carbonate } \\
\text { grains }^{\mathrm{a}}\end{array}$ & Others $^{b}$ \\
\hline \multicolumn{11}{|l|}{ Light beds } \\
\hline \multicolumn{11}{|l|}{$122-762 \mathrm{C}$ - } \\
\hline $\begin{array}{l}47 X-1,45 \\
48 X-1,90 \\
49 X-1,84\end{array}$ & 5.0 & & $\begin{array}{l}5.0 \\
2.0 \\
5.0\end{array}$ & & 1.0 & & $\begin{array}{r}15.0 \\
7.0 \\
80.0\end{array}$ & $\begin{array}{l}55.0 \\
80.0 \\
10.0\end{array}$ & $\begin{array}{l}25.0 \\
10.0\end{array}$ & \\
\hline $\begin{array}{l}49 X-1,84 \\
52 X-3,75\end{array}$ & $\begin{array}{l}2.0 \\
2.0\end{array}$ & $\begin{array}{l}1.0 \\
\operatorname{tr}\end{array}$ & $\begin{array}{l}5.0 \\
5.0\end{array}$ & 1.0 & & 2.0 & $\begin{array}{l}80.0 \\
13.0\end{array}$ & $\begin{array}{l}10.0 \\
75.0\end{array}$ & 2.0 & 2.0 \\
\hline $52 X-6,75$ & 2.0 & & 3.0 & & & & 16.0 & 67.0 & 2.0 & 10.0 \\
\hline $53 X-6,67$ & & & 5.0 & & & 2.0 & 10.0 & 82.0 & 1.0 & \\
\hline $54 X-1,86$ & $\mathrm{tr}$ & & 10.0 & & & & 7.0 & 76.0 & 7.0 & \\
\hline $54 X-5,84$ & 1.0 & & 5.0 & & & & 10.0 & 81.0 & 3.0 & \\
\hline $55 \mathrm{X}-2,22$ & $\operatorname{tr}$ & & 7.0 & & 1.0 & & 8.0 & 73.0 & 10.0 & 1.0 \\
\hline $56 \times-1,139$ & & & 15.0 & & & & 7.0 & 75.0 & 3.0 & \\
\hline $56 \times-3,110$ & & & 10.0 & & tr & $\operatorname{tr}$ & 13.0 & 73.0 & & 4.0 \\
\hline \multicolumn{11}{|l|}{ Dark beds } \\
\hline \multicolumn{11}{|l|}{$122-762 C$} \\
\hline $47 X-3,36$ & 3.0 & & 5.0 & & & & 15.0 & 62.0 & 15.0 & \\
\hline $47 X-3,66$ & 3.0 & & 7.0 & & & & 15.0 & 60.0 & 25.0 & \\
\hline $47 X-C C, 17$ & 2.0 & & 3.0 & & & & 5.0 & 80.0 & & \\
\hline $48 X-2,48$ & 2.0 & & 2.0 & & 5.0 & & 3.0 & 85.0 & 3.0 & \\
\hline $48 X-4,55$ & 3.0 & & 2.0 & & 3.0 & & 3.0 & 76.0 & 12.0 & \\
\hline $48 X-6,39$ & 4.0 & & 2.0 & & 2.0 & & 6.0 & 73.0 & 7.0 & 6.0 \\
\hline $48 X-C C, 38$ & 2.0 & & 1.0 & & 5.0 & & 10.0 & 77.0 & 5.0 & \\
\hline $49 \times-3,19$ & 4.0 & & 10.0 & & & & 8.0 & 59.0 & 15.0 & $2: 0$ \\
\hline $49 \times-5,92$ & & & 10.0 & & & & 10.0 & 80.0 & & \\
\hline $49 X-C C, 51$ & 5.0 & & 10.0 & & & & 5.0 & 65.0 & 15.0 & \\
\hline $52 \mathrm{X}-3,110$ & 9.0 & 2.0 & 7.0 & & & 2.0 & 7.0 & 70.0 & 3.0 & \\
\hline $52 X-6,105$ & 5.0 & $\operatorname{tr}$ & 13.0 & 2.0 & & 4.0 & 5.0 & 63.0 & 3.0 & 5.0 \\
\hline $53 \mathrm{X}-1,35$ & 2.0 & & 11.0 & 1.0 & 1.0 & & 7.0 & 50.0 & 28.0 & $\mathrm{tr}$ \\
\hline $53 X-2,55$ & $\operatorname{tr}$ & & 5.0 & 2.0 & 2.0 & & 12.0 & 65.0 & 12.0 & 2.0 \\
\hline $53 \mathrm{X}-3,122$ & 8.0 & & 8.0 & tr & & & 13.0 & 52.0 & 18.0 & 1.0 \\
\hline $54 X-3,67$ & & & 20.0 & & & tr & 8.0 & 69.0 & 3.0 & \\
\hline $55 \mathrm{X}-1,19$ & $\operatorname{tr}$ & & 27.0 & 1.0 & & & & 8.0 & 52.0 & 12.0 \\
\hline $55 X-3,86$ & $\operatorname{tr}$ & & 24.0 & & 1.0 & tr & 5.0 & 60.0 & 10.0 & \\
\hline $56 \mathrm{X}-5,50$ & & 20.0 & & & 1.0 & 5.0 & 71.0 & 3.0 & & \\
\hline
\end{tabular}

Note: Compiled from shipboard observations (Haq, von Rad, O'Connell, et al., 1990); tr = trace.

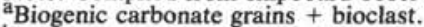

b Sponge spicules + fish remains + ostracodes. 


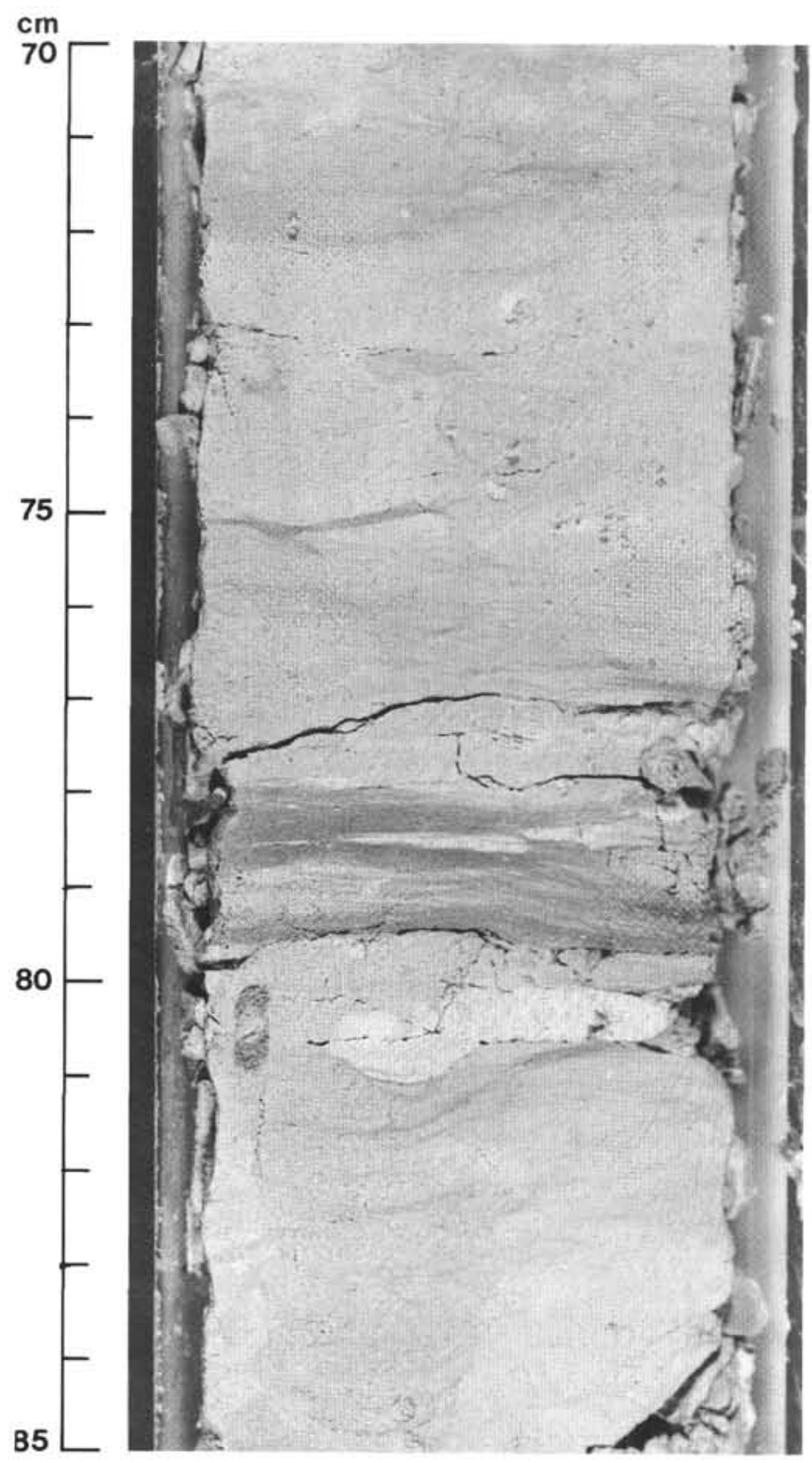

Figure 6. Diagenetic alteration of the sediments: small, thin seams cutting trace fossils (Sample 122-762C-56X-5, 70-85 cm).

the lower resolution of this spectrum. The peak in Figure 11A with a $30.7-\mathrm{cm}$ wavelength has its counterparts in both subsection spectra. In Figure 11B, there are some weaker peaks around the peak with a $30.7-\mathrm{cm}$ wavelength.

In the examination and interpretation of the power spectra, we shall first use the wavelength ratios of the frequency peaks to test the hypothesis that the cyclic sediments resulted from processes with Milankovitch frequencies. The wavelength ratios are examined and compared with the ratios of presentday periods of the orbital elements (Table 6). The reason for comparison with the ratio of present-day periods of the orbital elements is that the orbital element periods in the Cretaceous were not much shorter than present-day periods, which are more accurately calculated by Berger (1977). The use of ratios for interpretation is independent of absolute time and can avoid some errors brought in by sedimentation rate estimation. The ratio method was used by Molinie and Ogg (pers. comm., 1990) in analyzing Upper Jurassic and Lower Creta- ceous sedimentary cycles of the equatorial Pacific where stratigraphic constraints are uncertain. However, there are still some interfering factors in and limitations to this method. First, some of the ratios of orbital periodicities are very near. For example, the $\mathrm{O} / \mathrm{P} 1$ ratio $(2.15)$ is near the $\mathrm{E} 1 / \mathrm{O}$ ratio (2.31), and therefore a definite interpretation sometimes cannot be derived solely in the light of the wavelength ratio. Second, there are errors in the wavelength ratios which may result from the lack of precision in the position of the low-frequency peaks. Third, possible differential compaction between the more calcareous portion and the marly portion can also introduce errors to the ratio of two compacted sedimentary cycles. Furthermore, when a spectrum yields only one frequency peak, though a very rare situation, the ratio method cannot be applied. As the ratio comparison can be affected by the above factors, the time duration in thousands of years of frequency peaks converted from the estimated average sedimentation rate, which may also have errors, should also be examined. Mutual constraint of ratio comparison and time duration examination is expected to achieve more certain insight into the periodicities of the cyclic sequence.

In Figure 9A, the wavelength ratio of peak $a$ to $b$ is 4.36 , near the E1/P2 ratio (4.07, see Table 6), that of peak $a$ to $c$ is 5.18 , near the $\mathrm{E} 1 / \mathrm{P} 1$ and E2/P2 ratios (4.99 and 5.20, see Table 6), and that of peaks $b$ to $c$ is 1.19 , near the $\mathrm{P} 2 / \mathrm{P} 1$ ratio $(1.25$, see Table 6$)$. The time durations of peaks $a, b$, and $c$, from the estimated average sedimentation rate $(1.82 \mathrm{~cm} / \mathrm{ky})$, are 97.7 k.y., 22.4 k.y., and 18.8 k.y. These time durations are very near the modern periodicity of one of the eccentricity cycles $(94.9$ k.y.) and precession cycles ( 23.7 k.y. and 19.0 k.y.). Therefore, peak $a$ in Figure 9A (time series 1) is comparable to the short eccentricity cycle and peaks $b$ and $c$ to precession cycles. In this case the wavelength ratios fit very well with the time duration from the estimated sedimentation rate in interpreting the spectrum.

In Figure 10, the wavelength ratio of peak $a$ to $b$ is 2.20 , near the O/P1 ratio (2.15, see Table 6), and that of peak $a$ to $c$ is 2.40 , near the E1/O ratio (2.31, see Table 6$)$. The wavelength ratio of peak $b$ to $c$ is 1.09 , near the $\mathrm{P} 2 / \mathrm{P} 1$ ratio (1.25, see Table 6 ). The time duration from the estimated average sedimentation rate for peaks $a, b$, and $c$ are 46.2 k.y., 21.0 k.y., and $19.2 \mathrm{k} . \mathrm{y}$. The time duration for peak $a(46.2 \mathrm{k} . \mathrm{y}$.) is apparently much shorter that the periodicity of eccentricity. Combining both the wavelength ratio and the time duration estimated, peak $a$ is correlative to the obliquity cycle and peaks $b$ and $c$ to precession cycles.

In Figure 11A, the ratio of peak $a$ to $c(2.61)$ is between the $\mathrm{E} 2 / \mathrm{O}$ ratio (3.01, see Table 6$)$ and $\mathrm{E} 1 / \mathrm{O}$ ratio $(2.31$, see Table $6)$ and the ratio of peak $b$ to $c(2.31)$ is equal to the E1/O ratio (2.31, see Table 6). If interpreted just from the ratios, peaks $a$ and $b$ in this figure may have been related to the eccentricity cycle and peak $c$ to the obliquity cycle. However, the time durations from the estimated sedimentation rate for peaks $a$, $b$, and $c$ are 44.0 k.y., 38.9 k.y., and 16.9 k.y., respectively. The former two are much shorter than the periodicities of eccentricity and the latter is much shorter than the periodicity of obliquity. Also the positions of peaks $a$ and $b$ in this figure is comparable to those of peak $a$ in Figure 10, which is related to the obliquity cycle, and the position of peak $c$ in this figure is comparable to that of peaks $b$ and $c$ in Figure 10, which are related to precession cycles. Mainly based on the estimated time durations, we tentatively compare peaks $a$ and $b$ to the obliquity cycle and peak $c$ to the precession cycle. It may have been small fluctuations in sedimentation rate that produced two peaks out of one orbital element. This illustrates that interpretation of the power spectra of cyclic sediments just on the basis of wavelength ratio can sometimes be misleading. 
A
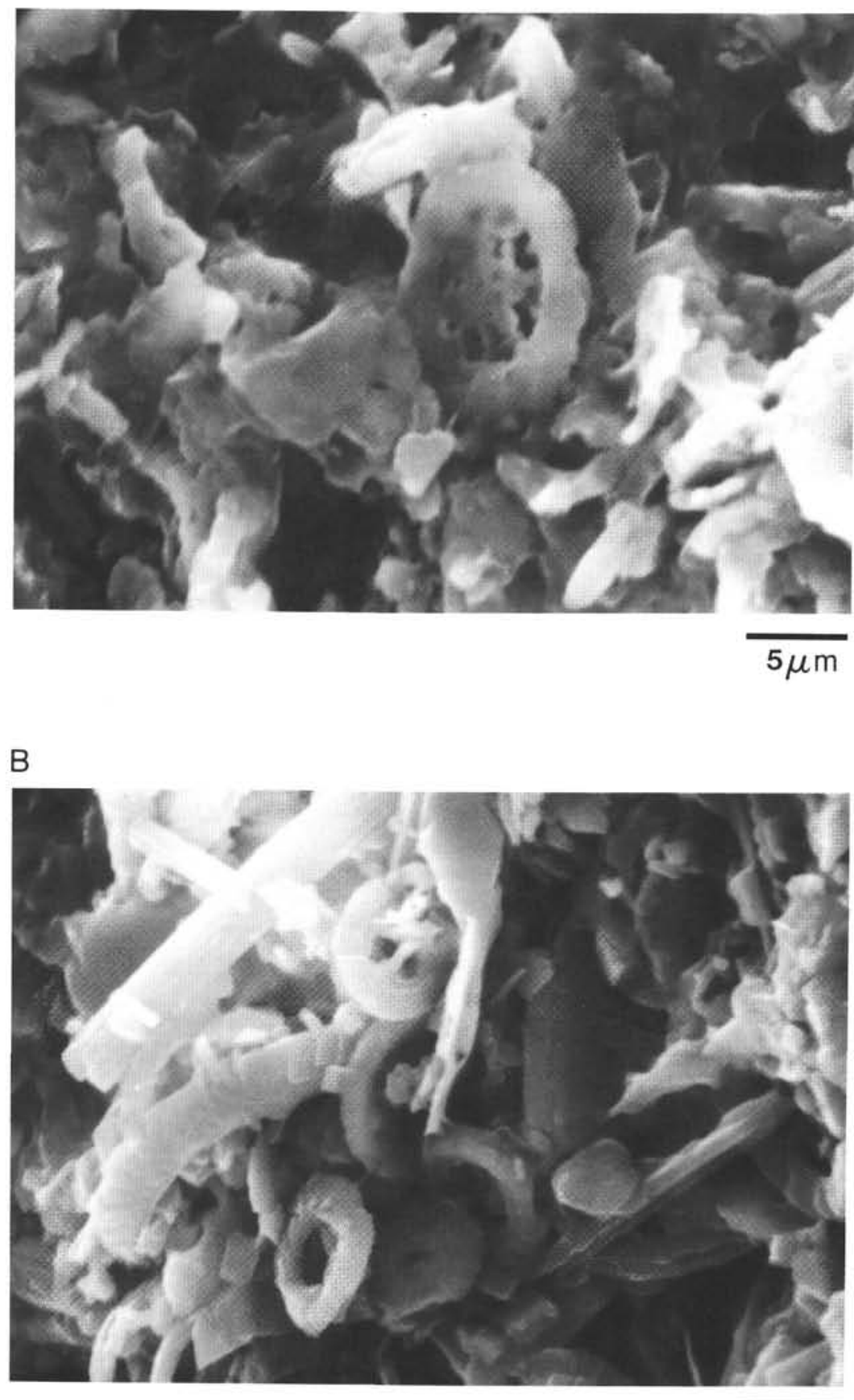

$5 \mu \mathrm{m}$

Figure 7. SEM photos of dark, clayey nannofossil chalk and light nannofossil chalk $(\times 5000)$. A. In the dark beds, the nannofossils are etched, with some secondary calcium carbonate deposition. Sample $122-762$ C-49X-3, 20-21 cm. B. In the light beds, the nannofossils are less dissolved, also with some secondary calcium carbonate deposition. The sediments are more porous. Sample $122-762 \mathrm{C}-49 \mathrm{X}-3,65-67 \mathrm{~cm}$.

We found that the wavelength ratios fit less well with the time durations downhole (from Figs. 9 to 11). This may be attributed to the increasing effect of differential compaction on the sediment column with depth, and also on the wavelength ratio method. The examination and interpretation of the power spectra with both wavelength ratios and time duration converted from the estimated average sedimentation rate demonstrate that the cyclic sequence from the Exmouth Plateau can be best related to the cyclic variations in orbital elements, rather than other processes. The color variation in the Upper Cretaceous cyclic sediments recovered from Hole $762 \mathrm{C}$ was most likely caused by long-term orbital variations. This is another example from Cretaceous strata support- 
Table 4. Physical properties of the light and dark beds in Cores 122-762C-48X through 122-762C-53X.

\begin{tabular}{lccccc}
\hline $\begin{array}{c}\text { Core, section, } \\
\text { interval }(\mathrm{cm})\end{array}$ & $\begin{array}{c}\text { Depth } \\
(\mathrm{mbsf})\end{array}$ & $\begin{array}{c}\text { Water } \\
\text { content } \\
(\%)\end{array}$ & $\begin{array}{c}\text { Wet-bulk } \\
\text { density } \\
\left(\mathrm{g} / \mathrm{cm}^{3}\right)\end{array}$ & $\begin{array}{c}\text { Grain } \\
\text { density } \\
\left(\mathrm{g} / \mathrm{cm}^{3}\right)\end{array}$ & $\begin{array}{c}\text { Porosity } \\
(\%)\end{array}$ \\
\hline Light beds & & & & & \\
122-762C- & & & & & \\
$48 X-1,90-92$ & 602.90 & 16.41 & 2.14 & 2.70 & 33.59 \\
$48 X-6,68-70$ & 610.18 & 20.97 & 2.03 & 2.70 & 41.62 \\
$49 X-1,9-11$ & 611.59 & 18.96 & 2.09 & 2.70 & 38.66 \\
$49 X-4,19-21$ & 616.19 & 21.39 & 2.04 & 2.71 & 42.54 \\
$50 X-4,94-96$ & 626.44 & 17.42 & 2.23 & 2.77 & 38.01 \\
$53 X-6,8-10$ & 657.08 & 23.31 & 2.02 & 2.68 & 45.90 \\
Mean & & 19.69 & 2.09 & 2.71 & 40.05 \\
& & & & & \\
Dark beds & & & & & \\
$122-762 C-$ & & & & & \\
$48 X-3,94-96$ & 605.94 & 16.91 & 2.13 & 2.68 & 35.09 \\
$48 X-5,55-57$ & 608.55 & 16.97 & 2.16 & 2.70 & 35.99 \\
$49 X-3,78-80$ & 615.28 & 12.54 & 2.29 & 2.72 & 28.04 \\
$49 X-5,132-134$ & 618.82 & 15.41 & 2.23 & 2.71 & 33.60 \\
$50 X-6,116-118$ & 629.66 & 19.98 & 2.19 & 2.82 & 42.75 \\
$51 X-2,66-68$ & 632.66 & 14.02 & 2.31 & 2.69 & 31.64 \\
$51 X-4,142-144$ & 636.42 & 18.33 & 2.21 & 2.70 & 39.49 \\
$51 X-5,142-144$ & 637.92 & 15.95 & 2.26 & 2.76 & 35.16 \\
$52 X-4,76-78$ & 645.26 & 18.01 & 2.19 & 2.73 & 38.54 \\
$53 X-2,20-22$ & 651.20 & 19.15 & 2.12 & 2.77 & 39.60 \\
Mean & & 16.73 & 2.21 & 2.73 & 36.02 \\
\hline
\end{tabular}

Note: Compiled from shipboard measurements (Haq, von Rad, O’Connell, et al., 1990).

Table 5. A summary on some important differences between the light and dark beds.

\begin{tabular}{|c|c|c|}
\hline & Light beds & Dark beds \\
\hline $\begin{array}{l}\text { Average thickness } \\
\text { and variance }(\mathrm{cm})\end{array}$ & $18 \pm 14$ & $22 \pm 18$ \\
\hline $\begin{array}{l}\mathrm{CaCO}_{3} \text { content and } \\
\text { variance }(\%)\end{array}$ & $86.99 \pm 3.17$ & $74.46 \pm 7.03$ \\
\hline $\begin{array}{l}\text { Clay content and } \\
\text { common type }\end{array}$ & $\begin{array}{l}\text { Low, more smectite than } \\
\text { koalite and illite }\end{array}$ & $\begin{array}{l}\text { High, more koalite and } \\
\text { illite than smectite }\end{array}$ \\
\hline $\begin{array}{l}\text { Quartz and } \\
\text { dolomite }\end{array}$ & Less & More \\
\hline Nannofossils & More & Less \\
\hline $\begin{array}{l}\text { Sponge spicules } \\
\text { and ostracodes }\end{array}$ & Less & More \\
\hline Physical properties & $\begin{array}{l}\text { Higher water content, } \\
\text { higher porosity }\end{array}$ & $\begin{array}{l}\text { Lower water content, } \\
\text { lower porosity }\end{array}$ \\
\hline
\end{tabular}

ing the Milankovitch theory. The obliquity, precession, and eccentricity signals are all found in this cyclic sequence, though the last is detected in only one of the time series. In the study by Golovchenko et al. (this volume) on the same interval, the histogram from thickness measurements in the core reveals that the most frequently occurring cycles are correlative to the obliquity and precession cycles. However, their analysis using the gamma-ray $\log$, whose vertical resolution is $0.3 \mathrm{~m}$ and not able to detect many of the color alternations, did not reveal lower frequencies whose frequency ratios and time duration are correlative to the eccentricity cycles. One possible reason for this may be that during the late Campanian to early Maestrichtian the eccentricity played a weak and unsteady role in causing cyclic sedimentation. In comparison to the other two orbital elements, eccentricity periodicities are less regular.

\section{MODEL FOR THE ORIGIN OF CYCLICITY}

With the establishment of Milankovitch-like cycles in Hole $762 \mathrm{C}$ and with the available sedimentological evidence, we now attempt to describe the origin of the sediment cyclicity.

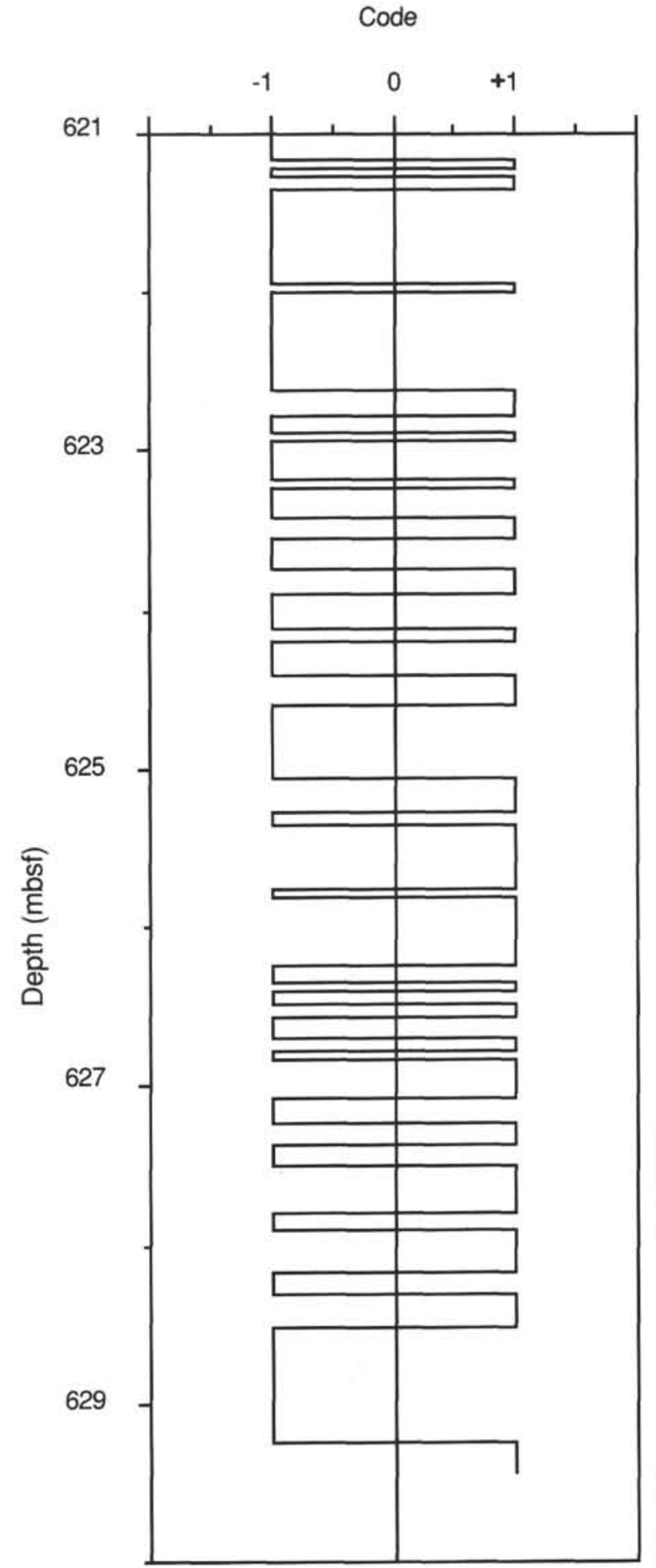

Figure 8. An example of the time series generated from the core inspection record (time series 2 ). The value +1 stands for light beds and -1 for dark beds. Sections 122-762C-50X-1 through 122-762C-50X-6.

We suggest that orbital variation may have controlled climatic change and thus induced the observed cyclic sedimentation on the Exmouth Plateau during the Late Cretaceous. Unfortunately, there are no calculated orbital and insolation changes in the Late Cretaceous. Previous studies have documented that the Late Cretaceous was globally warmer than present 


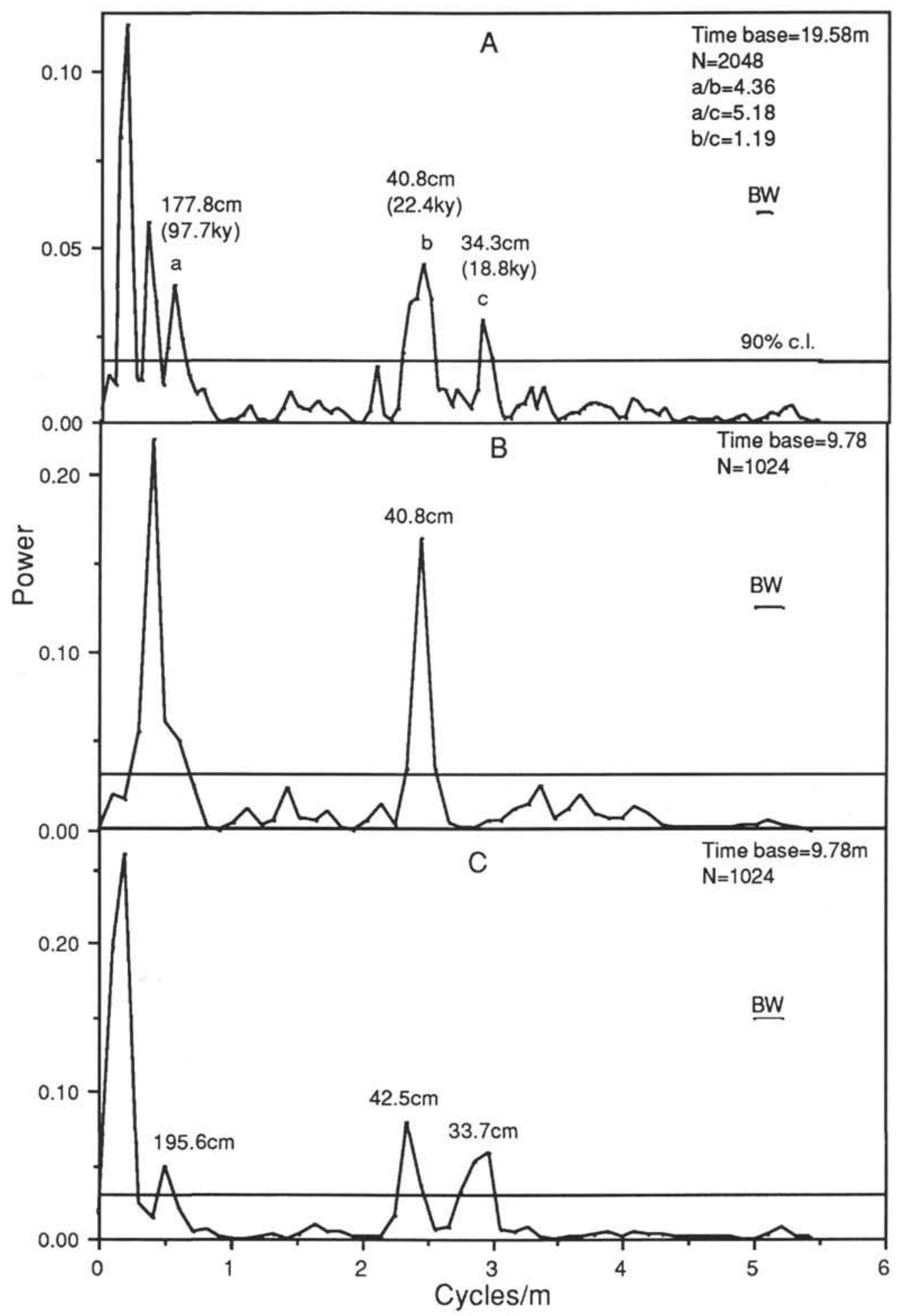

Figure 9. Power spectra for time series 1 . The resolution is indicated by the bandwidth (BW). "N" is the number of sampling points on the time series. The time base is the length of the time series being analyzed. The horizontal line is the $90 \%$ confidence level (c.l.) from Nowroozi (1967). The wavelength of the stable frequency peaks is marked in centimeters. Wavelengths in time (k.y.) from the average sedimentation rate of $1.82 \mathrm{~cm} / \mathrm{k} . \mathrm{y}$. are shown in parentheses. A. Whole-section spectrum for the entire time series 1. B. Subsection spectrum for the upper half of time series 1. C. Subsection spectrum for the lower half of time series 1. 
Table 6. Ratios of modern periods of the precession, obliquity, and eccentricity cycles to each other.

\begin{tabular}{lccccc}
\hline \multicolumn{1}{c}{ Periods (10 years) $^{3}$} & $\begin{array}{c}\mathrm{P} 1 \\
(19.0)\end{array}$ & $\begin{array}{c}\mathrm{P} 2 \\
(23.7)\end{array}$ & $\begin{array}{c}\mathrm{O} \\
(41.0)\end{array}$ & $\begin{array}{c}\mathrm{E} 1 \\
(94.9)\end{array}$ & $\begin{array}{c}\mathrm{E} 2 \\
(123.3)\end{array}$ \\
\hline $\begin{array}{l}\text { Ratio of precession (P1) to other } \\
\text { orbital elements }\end{array}$ & 1.00 & 0.80 & 0.46 & 0.20 & 0.15 \\
$\begin{array}{c}\text { Ratio of precession (P2) to other } \\
\text { orbital elements }\end{array}$ & 1.25 & 1.00 & 0.58 & 0.25 & 0.19 \\
$\begin{array}{c}\text { Ratio of obliquity (O) to other } \\
\text { orbital elements }\end{array}$ & 2.15 & 1.73 & 1.00 & 0.43 & 0.33 \\
$\begin{array}{c}\text { Ratio of eccentricity (E1) to other } \\
\text { orbital elements }\end{array}$ & 4.99 & 4.07 & 2.31 & 1.00 & 0.77 \\
$\begin{array}{c}\text { Ratio of eccentricity (E2) to other } \\
\text { orbital elements }\end{array}$ & 6.49 & 5.20 & 3.01 & 1.30 & 1.00 \\
\hline
\end{tabular}

Note: Orbital periods are according to Berger (1977).

and free of large-scale continental glaciation (Frakes, 1979; Barron, 1983). According to the Cretaceous plate tectonic reconstructions by Barron (1987), during Maestrichtian time the Australian continent, stretching west to east, was between $45^{\circ} \mathrm{S}$ to $65^{\circ} \mathrm{S}$. Ogg (pers. comm., 1990) gives an estimate of $53^{\circ} \mathrm{S}$ for the paleolatitude of the Exmouth region. In a latitudinal basin such as the Exmouth Plateau and with cyclic change in insolation caused by the obliquity and precession variations, we suggest a cyclic alternation of two different climatic regimes.

Given that the proportion of ocean and land areas is constant in a region, in higher insolation phases both the ocean and land received more energy from the sun. Because of the difference in the thermal inertia of water and land, the temperature increase in the ocean is much smaller than on the land. In this period, land-sea thermal contrast is great in summer, resulting in higher precipitation when more hot air rises over the continent to be replaced by moist, cooler marine air, but the summer-winter temperature difference is relatively small because the ocean serves as an efficient thermal reservoir and decreases the difference. In this phase, the climate has smaller seasonal contrast in temperature and is rainy. An "equable" warm and rainy climate favors the weathering of rocks, especially chemical weathering, and more clay minerals are formed on land. The high runoff because of higher precipitation increases summer terrestrial output of clay minerals (kaolinite and illite) and siliciclastic silt to the ocean. The rainy climate and high runoff may also lead to more precipitation than evaporation, which produces lower salinity surface water which may show seasonal variation. Warm surface water with varying and decreased salinity may not be suitable for most planktonic species to "bloom." Excessive sediments in suspension in the upper water column may also affect the development of a planktonic community. Total bioproductivity may be reduced in this phase for reasons of nutrient supply. The reduced equator-pole temperature contrasts during increased solar insolation (which affects higher latitudes more) may cause a decrease in wind strength, upwelling, and deep circulation. The nutrient supply may not be sufficient for marine plankton blooming. In such a climate, we consider that the dark beds with more clay were deposited at Site 762 .

In the low-insolation phase, the total energy from the sun is decreased. There is a smaller land-sea thermal contrast in the summer, making it less rainy in comparison with the summer in high-insolation phases. The seasonal contrasts (winter, summer) in temperature become pronounced as the function of the ocean as a thermal reservoir is reduced. The winter also becomes drier. Consequently, climate in the low-insolation phase is colder and drier. A colder, drier climate hinders the weathering of rock, especially chemical weathering, and fewer clay minerals are formed on land. The dilution of terrestrial input is reduced because of low precipitation and resulting low runoff. Planktonic species increase in abundance because freshwater influx causes surface waters to be less stratified, and the plankton experience greater nutrient replenishment from deeper levels in a period of increased wind strength and probably more upwelling. Under such a climate, the light dark beds were deposited at Site 762 .

The climatic mechanism considered to produce cyclic sedimentation at Site 762 is summarized in Figure 12, and is in accordance with the climatic modeling results of the Creta-

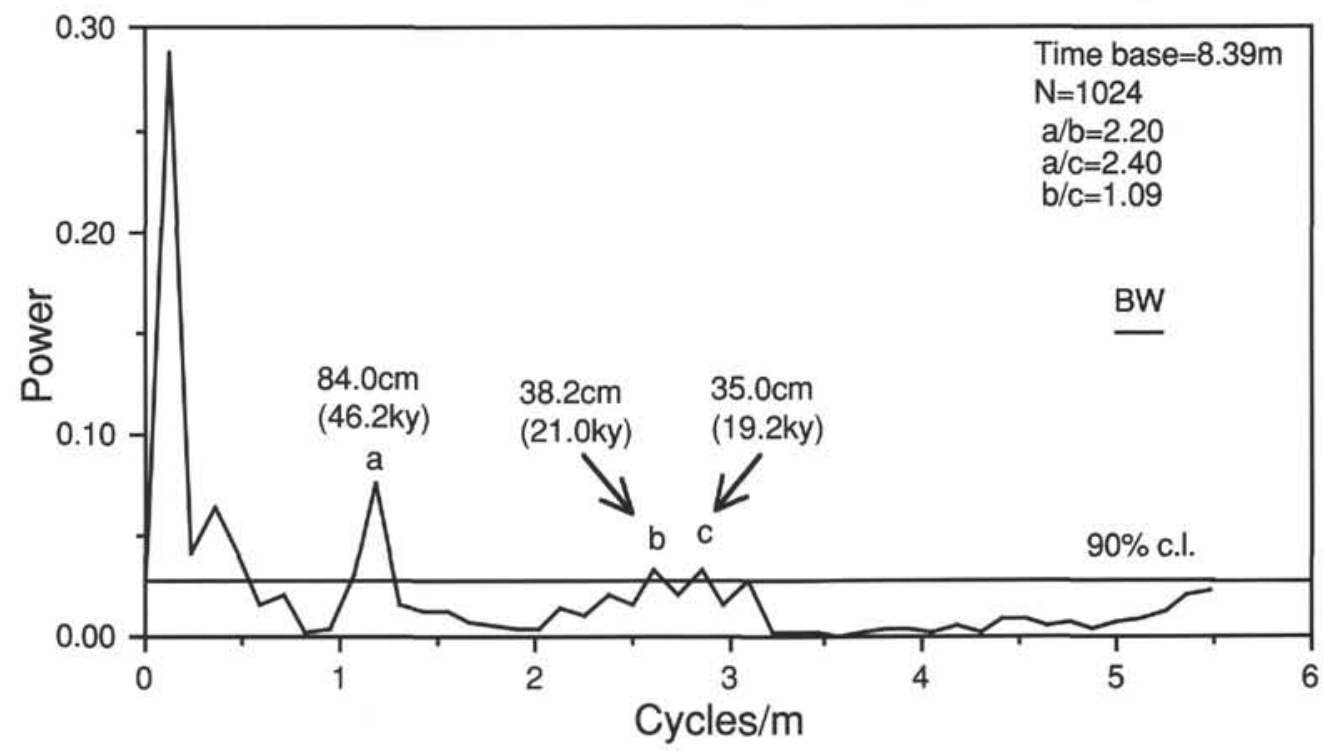

Figure 10. Power spectrum for time series 2 . The resolution is indicated by the bandwidth (BW). " $N$ " is the number of sampling points on the time series. The time base is the length of the time series. The horizontal line is the $90 \%$ confidence level (c.l.) from Nowroozi (1967). The wavelength of the stable frequency peaks is marked in centimeters. Wavelengths in time (k.y.) from the average sedimentation rate of $1.82 \mathrm{~cm} / \mathrm{k} . \mathrm{y}$. are shown in parentheses. 


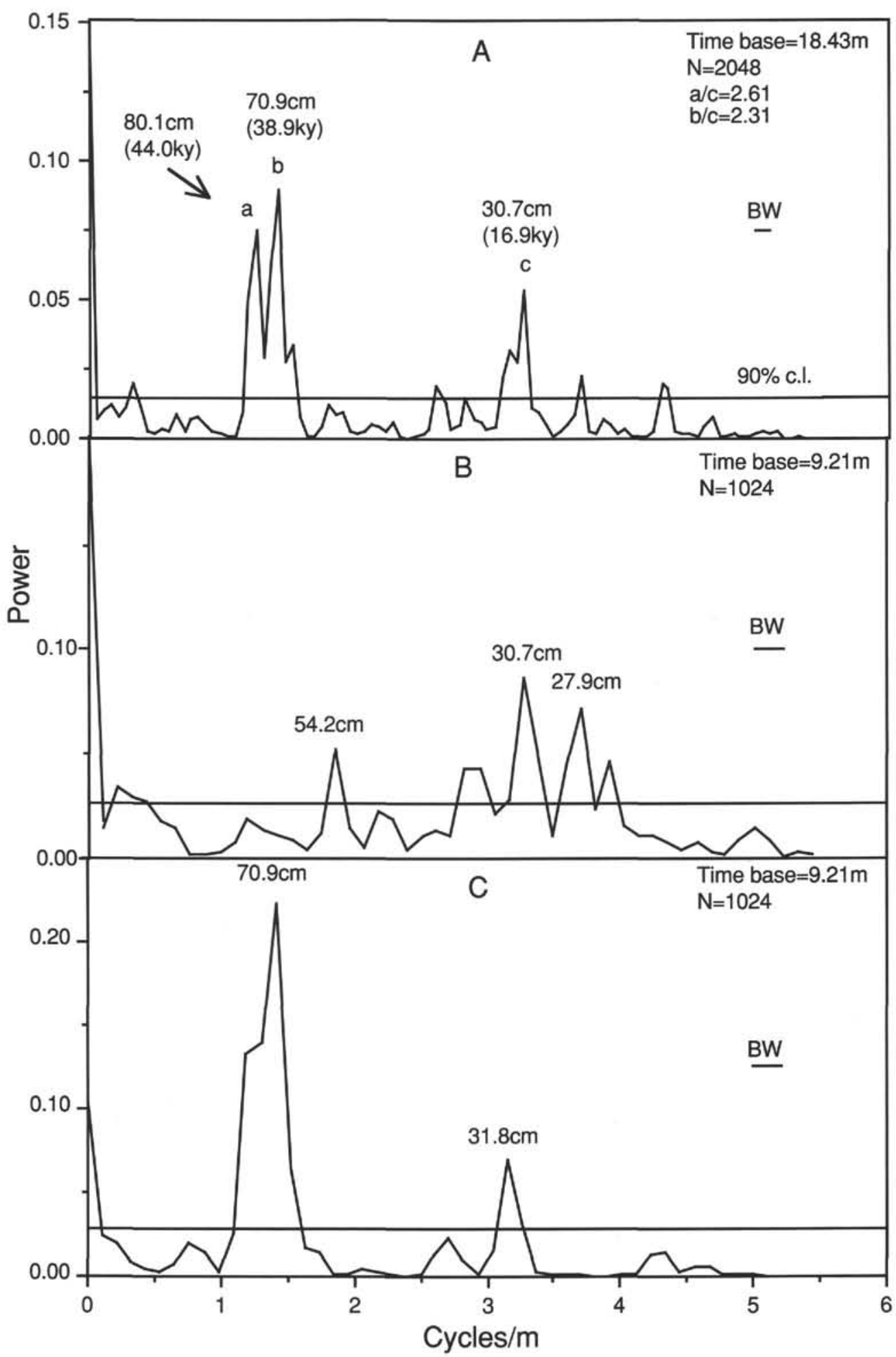

Figure 11. Power spectra for time series 3. The resolution is indicated by the bandwidth (BW). "N" is the number of sampling points on the time series. The time base is the length of the time series being analyzed. The horizontal line is the $90 \%$ confidence level (c.l.) from Nowroozi (1967). The wavelength of the stable frequency peaks is marked in centimeters. Wavelengths in time (k.y.) from the average sedimentation rate of $1.82 \mathrm{~cm} / \mathrm{k} . \mathrm{y}$. are shown in the parentheses. A. Whole-section spectrum for the entire time series 3 . B. Subsection spectrum for the upper half of time series 3. C. Subsection spectrum for the lower half of time series 3 . 
ceous by Oglesby and Park (1989). Although in their study an uncoupled climate model is employed, and only insolation variations caused by the precession cycle are considered, the results support our explanation for the climate-induced cyclic sedimentation on the Exmouth Plateau. Their results in South proto-Asia show that the January-July difference in land surface temperature is greater in a low-insolation phase than in a high-insolation phase, and in both January and July the specific humidity and precipitation is higher in a high-insolation phase than in a low-insolation phase.

In our model we have integrated the factors causing the variation in terrestrial material input and the change in bioproductivity. However, during the Late Cretaceous, under the control of orbital elements, the changes on land may have played a leading role in the cyclic sedimentation on the Exmouth Plateau. Changes in the ocean are secondary, as the planktonic microfauna are not only responding to water temperature changes that may not be very substantial owing to great thermal inertia, but also to input of terrestrial material and the surface salinity. Nutrient supply is another important factor for bioproductivity. As both dark and light beds are bioturbated in both climatic phases, the bottom water is well oxygenated. The oxygen levels in the bottom water may have been varying, but apparently never diminished enough to eliminate all bottom life. The fertility of the surface water may be mainly affected by influx of freshwater but not by upwelling or deep circulation, according to the paleo-water depth (550 m) estimated by Gradstein and von Rad (pers. comm., 1990) and the fact that the Exmouth Plateau is not far from the hinterland (terrestrial source).

The development of cycles in Pleistocene sediments involved changes in ice volume controlled by orbital variation (Pisias and Moore, 1981). Investigators of Upper Cretaceous cyclic sequences (R.O.C.C. Group, 1986) suggested that the sedimentary response to climatic forcing is variable. This study on the cyclic sediments recovered from Hole $762 \mathrm{C}$ not only supports the theory of an orbital origin of cyclicity in pelagic sediment, but also furnishes one possible mechanism for orbital variation controlling climatic change and hence cyclic sedimentation.

\section{DISCUSSION}

In the interval between 630.5 and 649.5 mbsf (time series 3) there are strong peaks most likely related to the obliquity and one less-strong peak possibly associated with precession (Fig. 11A). Between 621.0 and 630.0 mbsf (time series 2), the peak related to the obliquity is still strong (Fig. 10). In the upper interval 600.0 to 621 mbsf (time series 1), there are peaks possibly related to the eccentricity and precession cycles (Fig. 9A). However, according to both wavelength ratios and time duration from the average sedimentation rate, there is no peak in the whole-section spectrum (Fig. 9A), which can possibly be related to the obliquity cycle. This implies a transition from obliquity-controlled climatic variations to eccentricity-precession-controlled climatic variations. This also suggests that the response of the pelagic sedimentation system to the controlling effects of the long-term orbital variations was changing in the Late Cretaceous. Berger and Pestiaux (1984) suggest that insolation curves of lower latitudes mainly reflect the fluctuation in eccentricity and precession but in the curves of higher latitudes the obliquity cycle is clearly expressed; if so, this transform may be explained by a northward movement of the Indian plate in the late Campanian to early Maestrichtian. The sequence from the bottom of time series 3 to the top of time series 1 represents a time span of about $3 \mathrm{~m} . \mathrm{y}$., according to an average sedimentation rate of $1.82 \mathrm{~cm} / \mathrm{k} . \mathrm{y}$. Using a spreading rate of $2.0 \mathrm{~cm} / \mathrm{yr}$, the Indian Ocean plate could have moved $60 \mathrm{~km}$ north in this period. This seems insignificant for the Exmouth Plateau region to move into an eccentricityprecession-controlled low-latitude climate region from a obliquity-controlled high-latitude climate region if the cyclic sedimentation response to orbital variation were purely latitude dependent. Furthermore, according to Watts (1982), the separation of the Indian plate from the Antarctic plate and its large-scale northward motion occurred around the late $\mathrm{Pa}$ leocene, not in the Campanian and Maestrichtian. Another explanation may be that the falling sea level during the end of the late Campanian (Haq et al., 1987) caused some changes in the pattern of climatic variation. It appears that the response of the pelagic sedimentation system to the controlling effects of long-term orbital variations is complex and does not adhere to any simple relationship in the geological past. The study by Hagelberg and Pisias (1990) indicates that the climatic response to orbital forcing during the Pliocene is totally different from that during the Pleistocene.

In Figures 9, 10, and 11, the time durations converted from the average sedimentation rate for the peaks discussed are very near to the periodicities (modern values, compare with Table 6) of the orbital elements to which they are interpreted to be related. This may imply that a total 6-m.y. duration for the late Campanian to early Maestrichtian used for estimating the sedimentation rate is near the actual duration. However, there are some differences. For example, in the spectrum from time series 2 (Fig. 10) the peak associated with obliquity is longer ( 46.2 k.y.) than the obliquity periodicity (41.0 k.y.). This difference can be best explained by long-term variation in sedimentation rate. The actual sedimentation rate for this section (time series 2) could be higher than $1.82 \mathrm{~cm} / \mathrm{k} . \mathrm{y}$. (about $2.05 \mathrm{~cm} / \mathrm{k} . \mathrm{y}$.). The actual sedimentation rate for the other two sections (see Figs. 9 and 11) may not deviate much from the estimated average one $(1.82 \mathrm{~cm} / \mathrm{k} . \mathrm{y}$.). However, there are sufficient indications of sedimentation rate change in the intervals. For example, in Figure 11A there are two peaks whose time durations (44.0 k.y. and 38.9 k.y.) are both near obliquity periodicity ( $41 \mathrm{k} . \mathrm{y}$.$) . They are the product of one$ dominant obliquity cycle (41 k.y.). Two sedimentation rates $(1.95 \mathrm{~cm} / \mathrm{k} . \mathrm{y}$. and $1.73 \mathrm{~cm} / \mathrm{k} . \mathrm{y}$.$) are derived when the wave-$ lengths of these peaks are divided with the obliquity periodicity (41 k.y.). These two values may represent the fluctuating range of sedimentation rate in this interval (time series 3 ). In both Figures 9A and 10, two closely spaced peaks of about 20 $\mathrm{k} . \mathrm{y}$. in duration may be respectively related to the $23.7-\mathrm{k}$.y. and $19.0-\mathrm{k} . \mathrm{y}$. precession periodicities, but more likely they could be the result of just one of the two precession periods, separated by change in sedimentation rate.

The brown to green change in background hue at $603.5 \mathrm{mbsf}$ is believed to have been caused by a relative sea-level change (Haq, von Rad, O'Connell, et al., 1990) from highstand to lowstand. According to this explanation, the actively mixed ocean and the oxygenated water during the highstand period results in a brownish hue. This is not in accordance with the available evidence. In the whole interval we have studied, there is neither an obvious difference in the degree of bioturbation nor in the TOC content (see Table 2) in the sediments of different hue, indicating little change in the oxygen level in bottom water during the period when the sequence was deposited. In our view, the change in hue was more directly caused by the decreasing intensity of weathering on land. When the brownish-hued interval was deposited the global climate was warmer and more humid and the weathering products (clayey minerals, etc.) were brownish because of complete oxidation. When the greenishhued interval was deposited, the background climate became colder or at least less humid, and the weathering products (clayey minerals, etc.) were not fully oxidized and appeared 


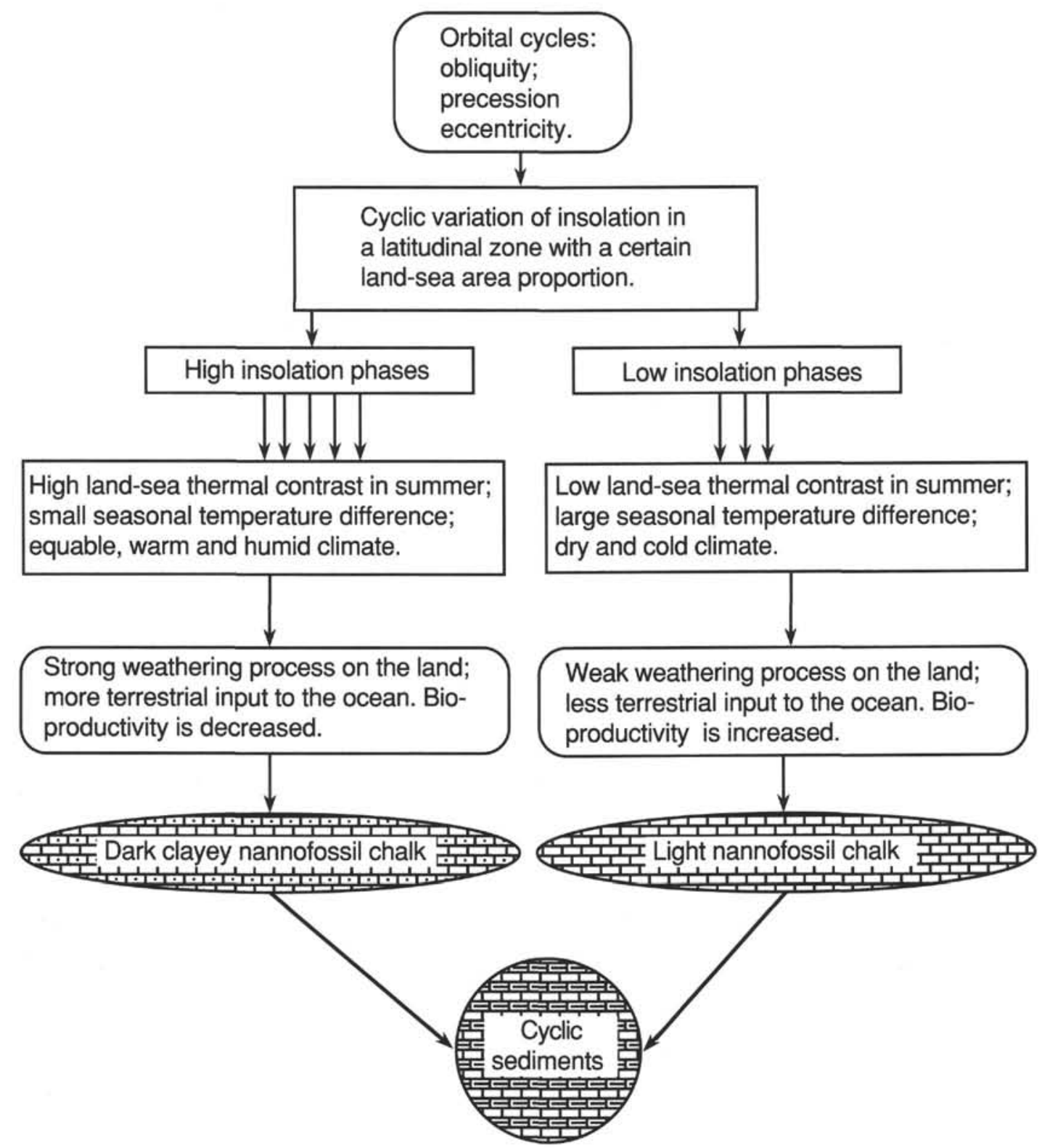

Figure 12. Suggested controlling mechanism of cyclic orbital variation for both the change of climate and the formation of cyclic sediments in the Late Cretaceous Indian Ocean (see text for detailed explanation).

greenish. It is also possible that the terrestrial input of iron occurred in a reduced state. This explanation is not in contradiction with the correlation between the hue change and the relative sea-level change, nor with the occurrence of pyrite in the greenish-hued interval. The relative sea-level change may have accompanied the overall hue change, but may not have necessarily been the final cause.

\section{CONCLUSIONS}

1. The cyclic color variation in the upper Campanian to lower Maestrichtian pelagic sediments from Site 762 is a depositional feature rather than a diagenetic result. Diagenetic processes at most enhanced the appearance of the cyclicity.

2. Spectral analysis has revealed that the predominant wavelength of the color cycles are $34-41 \mathrm{~cm}$ and 71-84 cm. With an average sedimentation rate of $1.82 \mathrm{~cm} / \mathrm{k} . \mathrm{y}$. , the time durations of the color cycles are around 41 k.y. and 21 k.y., correlative to the obliquity and precession periods and strongly suggesting an orbital origin for the cycles. A weak low-frequency signal correlative to the eccentricity cycle is found in the upper part of the sequence (time series 1). Therefore, the cyclic variations in the Upper Cretaceous strata from Hole $762 \mathrm{C}$ resulted from cyclic variations in orbital elements and we advocate that long-term orbital variations have been at work in the geological past.

3. During the Late Cretaceous, when there was no continental glaciation, cyclic sedimentation on the Exmouth Plateau of the eastern Indian Ocean was primarily caused by cyclic changes on land (soil formation and runoff) in response to orbitally-controlled insolation variations. Bioproductivity varied in phase with changes in terrestrial input, but no stagnant, oxygen-depleted bottom water was involved in the process. 
4. Spectral analysis indicates that the way in which orbital variations control cyclic sedimentation may vary with time. In the older part of the sequence (time series 2 and 3), there are strong obliquity signals with precession signals, but in the upper parts of the sequence (time series 1) there are only precession and eccentricity signals. A transition from obliquity-controlled climatic variations to eccentricity-precessioncontrolled climatic variations is implied. We suggest that the sedimentary response to the ultimate controlling force of orbital variations is neither necessarily constant through geological time in one place nor globally uniform at one time. Many factors can constrain the sedimentary response. The change from brownish hue to greenish hue in the sequence may indicate a major shift in the background climatic pattern, from very humid to less humid.

5. This investigation illustrates that building discrete time series on the basis of lithological inspection and calculating power spectra from such time series with the Walsh spectral analysis is an efficient, economical, and practical procedure for studying cyclic sediments. If subsurface materials are to be investigated, the constraints on this procedure are mainly the length of the interval and core preservation. Our experiences show that in examining and interpreting the power spectra it is more helpful to use both wavelength ratios and time durations from the estimated average sedimentation rate.

\section{ACKNOWLEDGMENTS}

We thank the Shipboard Scientific Party of Leg 122 for the cooperative scientific effort that made this study possible, G. Hampt for calcium carbonate measurements, and J. Tribble for XRD analysis. SEM examination was assisted by F. Thomas. This work benefited from discussions with Dr. R. Wilkens. Our manuscript was improved by comments and suggestions from Drs. F. M. Gradstein and J. G. Ogg. We are grateful to Drs. T. Herbert and T. Hagelberg for their critical review. Financial support for Z.H. and R.B. was provided by a Canadian NSERC Collaborative Special Project Grant and a University Research Grant from Imperial Oil (Canada), and for S.O. by USSAC.

\section{REFERENCES}

Arthur, M. A., Dean, W. E., Bottjer, D., and Scholle, P. A., 1984. Rhythmic bedding in Mesozoic-Cenozoic pelagic carbonate sequences: the primary and diagenetic origin of Milankovitch-like cycles. In Berger, A., Imbrie, J., Hays, J., Kukla, G., and Saltzman, B. (Eds.), Milankovitch and Climate (Pt. 1): Dordrecht (D. Reidel), 191-222.

Barron, E. J., 1983. A warm, equable Cretaceous: the nature of the problem. Earth Sci. Rev., 19:305-338.

Barron, E. J., 1987. Cretaceous plate tectonic reconstructions. Palaeogeogr., Palaeoclimatol., Palaeoecol., 59:3-29.

Barron, E. J., Arthur, M. A., and Kauffman, E. G., 1985. Cretaceous rhythmic bedding sequences: a plausible link between orbital variations and climate. Earth Planet. Sci. Lett., 72:327-340.

Beauchamp, K. G., 1975. Walsh Functions and Their Applications: New York (Academic Press).

Berger, A. L., 1977. Support for the astronomical theory of climatic change. Nature, 269:44-45.

Berger, A. L., and Loutre, M. F., 1989. Pre-Quaternary Milankovitch frequencies. Nature, 342:133.

Berger, A. L., and Pestiaux, P., 1984. Accuracy and stability of the Quaternary terrestrial insolation. In Berger, A., Imbrie, J., Hays, J., Kukla, G., and Saltzman, B. (Eds.), Milankovitch and Climate (Pt. 1): Dordrecht (D. Reidel), 83-111.

Bottjer, D. J., Arthur, M. A., Dean, W. E., Hattin, D. E., and Savrda, C. E., 1986. Rhythmic bedding produced in Cretaceous pelagic carbonate environments: sensitive recorders of climatic cycles. Paleoceanography, 1:467-481.
Cotillon, P., and Rio, M., 1984. Cyclic sedimentation in the Cretaceous of Deep Sea Drilling Project Sites 535 and 540 (Gulf of Mexico), 534 (Central Atlantic) and in the Vocontian Basin (France). In Buffler, R. T., Schlager, W., et al., Init. Repts. DSDP, 77: Washington (U.S. Govt. Printing Office), 339-376.

Darmedru, C., Cotillon, P., and Rio, M., 1982. Climatic and biological rhythms in marine pelagic realm: their relationships in Cretaceous alternating beds from the Vocontian Basin (SE France). Bull. Soc. Geol. Fr., 7:672-640.

Frakes, L., 1979. Climate through Geological Time: New York (Elsevier).

Hagelberg, T. K., and Pisias, N., 1990. Nonlinear response of Pliocene climate to orbital forcing: evidence from the Eastern Equatorial Pacific. Paleoceanography, 5:595-617.

Hallam, A., 1986. Origin of minor limestone-shale cycles: climatically induced or diagenetic? Geology, 14:609-612.

Haq, B. U., Hardenbol, J., and Vail, P. R., 1987. Chronology of fluctuating sea levels since the Triassic. Science, 235:1156-1167.

Haq, B. U., von Rad, U., O'Connell, S., et al., 1990. Proc. ODP, Init. Repts., 122: College Station, TX (Ocean Drilling Program).

Hart, M. B., 1987. Orbitally induced cycles in the chalk facies of the United Kingdom. Cretaceous Res., 8:335-348.

Hays, J. D., Imbrie, J., and Shackleton, N. J., 1976. Variations in the earth's orbit: pacemaker of the ice ages. Science, 194:1121-1132.

Herbert, T. D., and D'Hondt, S. L., 1990. Precession climate cyclicity in Late Cretaceous-Early Tertiary marine sediments: a high resolution chronometer of Cretaceous-Tertiary boundary events. Earth Planet. Sci. Lett., 99:263-275.

Herbert, T. D., and Fischer, A. G., 1986. Milankovitch climatic origin of Mid-Cretaceous black shale rhythms in Central Italy. Nature, 321:739-742.

Huffman, E.W.D., 1977. Performance of a new automatic carbon dioxide coulometer. Microchem. J., 22:567-573.

Imbrie, J., and Imbrie, J. Z., 1980. Modeling the climatic response to orbital variations. Science, 207:943-953.

Jarrard, R. D., and Arthur, M. A., 1989. Milankovitch Paleoceanographic cycles in geophysical logs from ODP Leg 105, Labrador Sea and Baffin Bay. In Srivastava, S. P., Arthur, M., Clement, B., et al., Proc. ODP, Sci. Results, 105: College Station, TX (Ocean Drilling Program), 757-772.

Kent, D. V., and Gradstein, F. M., 1985. A Cretaceous and Jurassic geochronology. Geol. Soc. Am. Bull., 96:1419-1427.

King, D. T., Jr., 1990. Upper Cretaceous marl-limestone sequences of Alabama: possible products of sea-level change, not climate forcing. Geology, 18:19-22.

Negi, J. G., and Tiwari, R. K., 1983. Matching long term periodicities of geomagnetic reversals and galactic motions of the solar system. Geophys. Res. Lett., 10:713-716.

Nowroozi, A. A., 1967. Table for Fischer's test of significance in harmonic analysis. Geophys. J. R. Astron. Soc., 12:512-520.

Ogg, J. G., 1987. Early Cretaceous magnetic polarity time scale and the magnetostratigraphy of Deep Sea Drilling Project Sites 603 and 534, Western central Atlantic. In van Hinte, J. E., Wise, S. W., Jr., et al., Init. Repts. DSDP, 93: Washington (U.S. Govt. Printing Office), 849-880.

Oglesby, R., and Park, J., 1989. The effect of precessional insolation changes on Cretaceous climate and cyclic sedimentation. J. Geophys. Res., 94:14973-14816.

Pisias, N. G., and Mix, A. C., 1988. Aliasing of the geologic record and the search for long-period Milankovitch cycles. Paleoceanography, 3:613-619.

Pisias, N. G., and Moore, T. C., Jr., 1981. The evolution of Pleistocene climate: a time series approach. Earth Planet. Sci. Lett., 52:450-458.

Research on Cretaceous Cycles (R.O.C.C.) Group (Arthur, M. A., Bottjer, D. J., Dean, W. E., Fischer, A. G., Hattin, D. E., Kauffman, E. G., Pratt, L. M., and Scholle, P. A.), 1986. Rhythmic bedding in Upper Cretaceous pelagic carbonate sequences: varying sedimentary response to climatic forcing. Geology, 14:153-156.

Schlumberger, 1989. Log Interpretation Principles/Applications: Houston, TX (Schlumberger Educ. Services).

Schwarzacher, W., 1987. The analysis and interpretation of stratification cycles. Paleoceanography, 2:79-95. 
Schwarzacher, W., and Fischer, A. G., 1982. Limestone-shale bedding and perturbation of the Earth's orbit. In Einsele, G., and Seilacher, A. (Eds.), Cyclic and Event Stratification: New York (Springer-Verlag), 72-95.

ten Kate, W. G., and Sprenger, A., 1989. On the periodicity in a calcilutite-marl succession (SE Spain). Cretaceous Res., 10:1-31.

Watts, A. B., 1982. Tectonic subsidence, flexure and global changes of sea level. Nature, 297:469-474.

Weedon, G. P., 1986. Hemipelagic shelf sedimentation and climatic cycles: the basal Jurassic (Blue Lias) of South Britain. Earth Planet. Sci. Lett., 76:321-335.

Date of initial receipt: 28 May 1990

Date of acceptance: 29 January 1991

Ms 122B-148

\section{APPENDIX}

Calcium Carbonate Measurement With Coulometric Method

Samples were dried in either a $100^{\circ} \mathrm{C}$ oven or in a freeze drier, powdered, weighed to the nearest milligram, and analyzed for calcium carbonate using a Coulometrics $\mathrm{CO}_{2}$ Coulometer. The Coulometer has an accuracy of $0.15 \%( \pm 0.2 \mathrm{mg})$.

The Coulometric titration technique measures all of the $\mathrm{CO}_{2}$ that is liberated by acidifying and heating sediment samples in a closed system. To do this, the powdered samples are placed in a test tube which is attached to the Coulometer, placed in a heat shield, and $2 \mathrm{H}$ $\mathrm{HCl}$ is pumped into the test tube. The liberated $\mathrm{CO}_{2}$ is transferred through scrubbers (solutions to remove interfering substance) by a $\mathrm{CO}_{2}$-free gas into an absorption cell where it is titrated through coulometric means. The absorption cell contains an aqueous medium of thanolamine and a coulometric indicator. The interaction of the $\mathrm{CO}_{2}$ and the cell solution creates a titratable acid. The coulometer forms a base electrically and titrates to an endpoint determined by the optical transmission of the indicator (Huffman, 1977). The pulse output was scaled and fed to a counter in terms of $\mathrm{CO}_{2}$. A stable Coulometer reading indicates that all of the $\mathrm{CO}_{2}$ has been evolved and titrated. The reading was recorded and the results were calculated in micrograms carbon and $\mathrm{CaCO}_{3}$ (wt $\% \mathrm{CaCO}_{3}=w t \% \mathrm{C}_{\text {inorganic }}$ $\times$ 8.334). This method of calculation assumes that all of the $\mathrm{CO}_{2}$ is liberated from calcium carbonate, but some of the $\mathrm{CO}_{2}$ may be liberated from dolomite, siderite, or other carbonate minerals. 